\title{
A Mathematical Theory for Numerical Treatment of Nonlinear Two-Point Boundary Value Problems
}

\author{
Tetsuro Yамамото and Shin'ichi Oishi
}

\author{
School of Science and Engineering, Waseda University, \\ Okubo 3-4-1, Shinjuku-ku, Tokyo 169-8555, Japan \\ E-mail: tetsuro.yamamoto@aoni.waseda.jp \\ E-mail: oishi@waseda.jp
}

Received August 26, 2004

Revised May 2, 2005

\begin{abstract}
This paper gives a unified mathematical theory for numerical treatment of two-point boundary value problems of the form $-\left(p(x) u^{\prime}\right)^{\prime}+f\left(x, u, u^{\prime}\right)=0, a \leq x \leq b, \alpha_{0} u(a)-$ $\alpha_{1} u^{\prime}(a)=\alpha, \beta_{0} u(b)+\beta_{1} u^{\prime}(b)=\beta, \alpha_{0}, \alpha_{1}, \beta_{0}, \beta_{1} \geq 0, \alpha_{0}+\alpha_{1}>0, \beta_{0}+\beta_{1}>0$, $\alpha_{0}+\beta_{0}>0$. Firstly, a unique existence of solution is shown with the use of the Schauder fixed point theorem, which improves Keller's result [6]. Next, a new discrete boundary value problem with arbitrary nodes is proposed. The unique existence of solution for the problem is also proved by using the Brouwer theorem, which extends some results in Keller [6] and Ortega-Rheinboldt [10]. Furthermore, it is shown that, under some assumptions on $p$ and $f$, the solution for the discrete problem has the second order accuracy $O\left(h^{2}\right)$, where $h$ denotes the maximum mesh size. Finally, observations are given.
\end{abstract}

Key words: two-point boundary value problems, existence of solution, error estimate, fixed point theorems, finite difference methods

\section{Introduction}

Two-point boundary value problems of the form

$$
\begin{gathered}
-\frac{d}{d x}\left(p(x) \frac{d u}{d x}\right)+f\left(x, u, \frac{d u}{d x}\right)=0, a \leq x \leq b \\
B_{1}(u) \equiv \alpha_{0} u(a)-\alpha_{1} u^{\prime}(a)=\alpha, \\
B_{2}(u) \equiv \beta_{0} u(b)+\beta_{1} u^{\prime}(b)=\beta,
\end{gathered}
$$

where $\alpha_{i}, \beta_{i}, i=1,2, \alpha$ and $\beta$ are constants, arise in many areas of applied science. They are solved numerically on computers by discretizing (1.1)-(1.3) at some nodes $\left\{x_{i}\right\}$ :

$$
a=x_{0}<x_{1}<\cdots<x_{n}<x_{n+1}=b .
$$

Throughout this paper, we assume $p(x)>0$ in $[a, b]$,

$$
\begin{gathered}
\alpha_{0} \geq 0, \alpha_{1} \geq 0, \alpha_{0}+\alpha_{1}>0 \\
\beta_{0} \geq 0, \beta_{1} \geq 0, \beta_{0}+\beta_{1}>0, \\
\alpha_{0}+\beta_{0}>0
\end{gathered}
$$


and put

$$
\begin{aligned}
& \mathscr{D}=\left\{u \in C^{2}[a, b] \mid B_{1}(u)=B_{2}(u)=0\right\} . \\
& h_{i}=x_{i}-x_{i-1}, i=1,2, \ldots, n+1, h=\max _{i} h_{i}
\end{aligned}
$$

Our main concerns are the following three subjects.

1. To guarantee existence and uniqueness of solution for (1.1)-(1.3).

2. To guarantee existence and uniqueness of solution for the discretized problem $\boldsymbol{F}(\boldsymbol{U})=\mathbf{0}$, where $\boldsymbol{U}=\left(U_{0}, U_{1}, \ldots, U_{n+1}\right)^{t}$ and each $U_{i}$ denotes an approximation for the exact value $u_{i}=u\left(x_{i}\right)$.

3. To estimate the errors for $\left|u_{i}-U_{i}\right|, i=0,1,2, \ldots, n+1$.

Concerning the first subject, let $p(x) \in C^{1}[a, b]$, and $f(x, u, v)$ be continuous on $\mathscr{R}=[a, b] \times \mathbb{R} \times \mathbb{R}$ and satisfy there a uniform Lipschitz condition in $u$ and $v$. Furthermore, let $f$ have continuous derivatives on $\mathscr{R}$ which satisfy

$$
\frac{\partial f}{\partial u}>0
$$

and

$$
\left|\frac{\partial f}{\partial v}\right| \leq M
$$

for some positive constant $M$. Under these assumptions, Keller ([6], Theorem 1.2.2) proved that the problem (1.1)-(1.3) has a unique solution $u \in C^{2}[a, b]$. If $p=1$, $f=f(x, u)$ and the conditions (1.2) and (1.3) are of Dirichlet's type $\left(\alpha_{0}=\beta_{0}=\right.$ $\left.1, \alpha_{1}=\beta_{1}=0\right)$, then it is also known that (1.1)-(1.3) has a unique solution if

$$
0 \leq \frac{\partial f}{\partial u} \leq K
$$

for some positive constant $K$ (Henrici [5]) or, if $[a, b]=[0,1], \alpha=\beta=0$ and

$$
\inf _{[a, b] \times \mathbb{R}} \frac{\partial f}{\partial u}>-\pi^{2} \quad(\text { Lees }[8]) .
$$

However, in the case $f=f(x, u, v)$, there is no existence and uniqueness result of solution for (1.1)-(1.3) under the condition $f_{u} \geq 0$.

Concerning the second subject, some results for the case $f=f(x, u)$ can be found in Ortega-Rheinboldt [10]. For example, if $\Lambda$ is an $M$-matrix and $\Phi$ : $\mathbb{R}^{n+2} \rightarrow \mathbb{R}^{n+2}$ is a continuous, diagonal and isotone mapping, then the system of $n+2$ equations

$$
\Lambda \boldsymbol{U}+\Phi(\boldsymbol{U})=\mathbf{0}
$$

has a unique solution. Furthermore, in the case $f=f(x, u, v)$, Keller ([6], Theorem 3.2.2) proved that, if $p(x)=1$ and the conditions (1.2) and (1.3) are of 
Dirichlet's type, then the system of the standard finite difference approximations with uniform nodes $\left(h=h_{i} \quad \forall i\right)$ applied to (1.1)

$$
\begin{gathered}
\frac{1}{h^{2}}\left(-U_{i-1}+2 U_{i}-U_{i+1}\right)+f\left(x_{i}, U_{i}, \frac{U_{i+1}-U_{i-1}}{2 h}\right)=0,1 \leq i \leq n \\
U_{0}=\alpha, \quad U_{n+1}=\beta
\end{gathered}
$$

has a unique solution for sufficently small $h$, provided that $f(x, u, v)$ has continuous derivatives on $\mathscr{R}$ which satisfy (1.10) and

$$
0<K_{*} \leq \frac{\partial f}{\partial u} \leq K
$$

for some positive constants $K$ and $K_{*}$. It seems to the authors, however, that no mention about existence and uniqueness of solution for a discretized system with nonuniform nodes has also been made under the condition $\frac{\partial f}{\partial u} \geq 0$.

Concerning the third subject, for the case where (1.1) is of the Sturm-Liouville type, Tikhonov-Samarskii [11] first pointed out that the usual finite difference approximation applied to $(1.1)-(1.3)$ has the second order accuracy $O\left(h^{2}\right)$ with any nodes (1.4) under the assumption

$$
0<C_{1} \leq \frac{h_{i+1}}{h_{i}} \leq C_{2} \quad \forall i,
$$

where $C_{1}, C_{2}$ are positive constants independent of the nodes. Since the local truncation error $\tau_{i}$ at $x_{i}$ is $O(h)$ if $h_{i} \neq h_{i+1}$, this indicates that the order of the local truncation error is not a true measure for the error of numerical solution. Later, in 1985, Hoog-Jackett [4] proved the same result without assuming (1.13), provided that $f$ is linear and $u \in C^{4}[a, b]$. More general discussion is given in ManteuffelWhite, Jr. [9] and Kreiss-Manteuffel-Swartz-Wendroff-White, Jr. [7]. Among others, in the latter paper, this phenomenon is called "supra-convergence".

In this paper, replacing the condition in (1.9) by a weaker one $\frac{\partial f}{\partial u} \geq 0$, we shall treat the above three subjects. First, in $\S 2$, with the use of the Schauder fixed point theorem, we shall show that the problem (1.1)-(1.3) has a unique solution $u \in C^{2}[a, b]$ if $p \in C^{1}[a, b], 0 \leq f_{u} \leq K$ and $\left|f_{v}\right| \leq M$ on $\mathscr{R}=[a, b] \times \mathbb{R} \times \mathbb{R}$, which improves Keller's result. It should be noted here (cf. Remark 5.1) that, if $f=f(x, u)$, then the conditions $0 \leq f_{u} \leq K$ can be replaced by $f_{u} \geq 0$. The existence of a constant $K$ is not necessary. Hence our result improves and generalizes Henrici's result. Next, in $\S 3$, we shall propose an approximate method with arbitrary nodes (1.4). The method solves a system of $n+2$ equations

$$
F(\boldsymbol{U}) \equiv H A \boldsymbol{U}+\tilde{\boldsymbol{f}}(\boldsymbol{U})=0
$$

where $H$ is a diagonal matrix, $A$ is a tridiagonal matrix whose elements include

$$
\int_{x_{i-1}}^{x_{i}} \frac{d t}{p(t)}, \quad 1 \leq i \leq n+1
$$


and $\widetilde{\boldsymbol{f}}(\boldsymbol{U})$ is defined in (3.3). As is shown in $\S 3$, the matrix $A$ has the distinguished feature that $A^{-1}=\left(G\left(x_{i}, x_{j}\right)\right)$, where $G(x, \xi)$ denotes the Green function for the operator $\mathscr{L}=-\frac{d}{d x}\left(p \frac{d}{d x}[\quad]\right): \mathscr{D} \rightarrow C[a, b]$ and $\mathscr{D}$ is defined by (1.8). If we approximate $(1.15)$ by the midpoint rule and the boundary conditions by the fictitious node method, then the equation (1.14) reduces to the standard finite difference equation.

In $\S 4$, with the use of the Brouwer fixed point theorem, we shall prove that (1.14) has a unique solution for sufficiently small $h$, provided that $p \in C^{1}[a, b]$, $f_{u} \geq 0$ and $\left|f_{v}\right| \leq M$. The result generalizes known results in Keller [6], OrtegaRheinboldt [10], etc.

Furthermore, in $\S 5$, we shall extend Hoog-Jackett's argument to the case where $f=f\left(x, u, u^{\prime}\right)$ is nonlinear and prove

$$
u_{i}-U_{i}=O\left(h^{2}\right) \quad \forall i
$$

provided that $p \in C^{2,1}[a, b]$ and $f$ satisfies some smoothness conditions in $x, u$ and $u^{\prime}$ on $\mathscr{R}$.

Finally, in $\S 6$, observations will be given.

\section{Existence of Solution for (1.1)-(1.3)}

In this section, we shall prove the existence and uniqueness of solution for the problem (1.1)-(1.3). We first remark that if we take a function $v(x)=\lambda x^{2}+\mu x+\nu$ where coefficients $\lambda, \mu, \nu$ are determined so that $B_{1}(v)=\alpha$ and $B_{2}(v)=\beta$, then $w=u-v$ satisfies $B_{1}(w)=B_{2}(w)=0$ and $w$ satisfies a differential equation

$$
-\frac{d}{d x}\left(p \frac{d w}{d x}\right)+\widehat{f}\left(x, w, \frac{d w}{d x}\right)=0
$$

with $\widehat{f}=-\frac{d}{d x}\left(p \frac{d v}{d x}\right)+f\left(x, w+v, \frac{d w}{d x}+\frac{d v}{d x}\right)$, which is of the form (1.1). Hence, the unique existence of solution for the equation (1.1) in $\mathscr{D}$ implies that for the problem (1.1)-(1.3) in $C^{2}[a, b]$.

Before proving the existence theorem of solution for (1.1)-(1.3), we state some elementary lemmas whose proofs will be given for the sake of completeness.

LEMMA 2.1. Let

$$
\mathscr{L} u=-p(x) \frac{d^{2} u}{d x^{2}}+q(x) \frac{d u}{d x}+r(x) u
$$

with $p \in C^{1}[a, b], q, r \in C[a, b], p>0$ and $r \geq 0$ in $[a, b]$. Then, the Green function exists for $(\mathscr{L}, \mathscr{D})$. let

Proof. It suffices to show that $\mathscr{L} u=0$ and $u \in \mathscr{D}$ imply $u=0$. To show this,

$$
P(x)=e^{-\int_{a}^{x} \frac{q(t)}{p(t)} d t}, \quad R(x)=P(x) \frac{r(x)}{p(x)} \text { and } \widetilde{\mathscr{L}}=\frac{P(x)}{p(x)} \mathscr{L} .
$$


Then $P(x)>0, R(x) \geq 0$ and $\mathscr{L} u=0$ is equivalent to

$$
\widetilde{\mathscr{L}} u=-\frac{d}{d x}\left(P(x) \frac{d u}{d x}\right)+R(x) u=0 .
$$

Hence

$$
(\widetilde{\mathscr{L}} u, u)=\int_{a}^{b}\left\{-\frac{d}{d x}\left(P(x) \frac{d u}{d x}\right)+R(x) u\right\} u d x=0
$$

or

$$
\Phi+\int_{a}^{b}\left\{P(x)\left(\frac{d u}{d x}\right)^{2}+R(x) u^{2}\right\} d x=0
$$

with $\Phi=P(a) u^{\prime}(a) u(a)-P(b) u^{\prime}(b) u(b)$.

If $\alpha_{0}>0$, then

$$
P(a) u^{\prime}(a) u(a)=P(a) \frac{\alpha_{1}}{\alpha_{0}} u^{\prime}(a)^{2} \geq 0
$$

and

$$
-P(b) u^{\prime}(b) u(b)= \begin{cases}P(b) \frac{\beta_{1}}{\beta_{0}} u^{\prime}(b)^{2} & \left(\beta_{0}>0\right) \\ 0 & \left(\beta_{0}=0\right)\end{cases}
$$

Hence $\Phi \geq 0$ and we have from (2.1)

$$
\Phi=0 \quad \text { and } \quad \frac{d u}{d x} \equiv 0 .
$$

We thus obtain that $u$ is a constant on $[a, b]$. But, $u \in \mathscr{D}$ then implies

$$
B_{1}(u)=\alpha_{0} u(a)=0
$$

and we must have $u(a)=0$, i.e., $u \equiv 0$. Similarly, if $\beta_{0}>0$, then we obtain $u(b)=0$ and $u \equiv 0$. Consequently, by (1.7), we have $u \equiv 0$ on $[a, b]$.

Q.E.D.

Lemma 2.2. Let $p(x) \in C^{1}[a, b], p>0$ in $[a, b]$ and

$$
\mathscr{L} u=-\frac{d}{d x}\left(p(x) \frac{d u}{d x}\right), \quad u \in \mathscr{D} .
$$

Then the Green function $G(x, \xi)$ for $(\mathscr{L}, \mathscr{D})$ is given by

$$
\begin{aligned}
G(x, \xi) & = \begin{cases}\frac{1}{p(a) p(b) \delta}\left(\alpha_{1}+\alpha_{0} p(a) \int_{a}^{x} \frac{d t}{p(t)}\right)\left(\beta_{1}+\beta_{0} p(b) \int_{\xi}^{b} \frac{d t}{p(t)}\right) & (x \leq \xi) \\
\frac{1}{p(a) p(b) \delta}\left(\alpha_{1}+\alpha_{0} p(a) \int_{a}^{\xi} \frac{d t}{p(t)}\right)\left(\beta_{1}+\beta_{0} p(b) \int_{x}^{b} \frac{d t}{p(t)}\right) & (x \geq \xi)\end{cases} \\
\leq & G(x, x),
\end{aligned}
$$


where

$$
\delta=\alpha_{0}\left(\beta_{0} \int_{a}^{b} \frac{d t}{p(t)}+\frac{\beta_{1}}{p(b)}\right)+\frac{\alpha_{1} \beta_{0}}{p(a)}>0
$$

Proof. Let

$$
\begin{aligned}
& \varphi_{1}(x)=-\alpha_{1}-\alpha_{0} p(a) \int_{a}^{x} \frac{d t}{p(t)} \\
& \varphi_{2}(x)=\beta_{1}-\beta_{0} p(b) \int_{b}^{x} \frac{d t}{p(t)} .
\end{aligned}
$$

Then $\mathscr{L} \varphi_{i}=0$ and $B_{i}\left(\varphi_{i}\right)=0, i=1,2$. Hence, by the elementary theory on ordinary differential equations, we have

$$
G(x, \xi)= \begin{cases}\frac{\varphi_{1}(x) \varphi_{2}(\xi)}{-p(a) W\left(\varphi_{1}, \varphi_{2}\right)(a)} & (x \leq \xi) \\ \frac{\varphi_{1}(\xi) \varphi_{2}(x)}{-p(a) W\left(\varphi_{1}, \varphi_{2}\right)(a)} & (x \geq \xi),\end{cases}
$$

where $W\left(\varphi_{1}, \varphi_{2}\right)(x)$ denotes the Wronskian determinant on $\varphi_{1}$ and $\varphi_{2}$. Lemma 2.2 follows from this.

Q.E.D.

The following Lemmas 2.3 and 2.4 are analogues of the well known results on $M$-matrices.

Lemma 2.3. Assume that $p(x) \in C^{1}[a, b], r(x) \in C^{1}[a, b], p(x)>0$ and $r(x) \geq 0$. Let

$$
\mathscr{L}_{r} u=-\frac{d}{d x}\left(p(x) \frac{d u}{d x}\right)+r(x) u
$$

and $G_{r}(x, \xi)$ be the Green function for $\left(\mathscr{L}_{r}, \mathscr{D}\right)$. Then $G_{r}(x, \xi)>0$ for all $x, \xi \in$ $(a, b)$.

Proof. Let $\varphi_{1}(x)$ and $\varphi_{2}(x)$ be the solutions of the following initial value problems, respectively:

$$
\begin{array}{ll}
\mathscr{L}_{r} \varphi_{1}=0, \quad \varphi_{1}(a)=\alpha_{1}(\geq 0), \quad \varphi_{1}^{\prime}(a)=\alpha_{0}(\geq 0) \\
\mathscr{L}_{r} \varphi_{2}=0, \quad \varphi_{2}(b)=\beta_{1}(\geq 0), \quad \varphi_{2}^{\prime}(b)=-\beta_{0}(\leq 0) .
\end{array}
$$

Then $B_{i}\left(\varphi_{i}\right)=0, i=1,2$. The Green function $G_{r}(x, \xi)$ is given by

$$
G_{r}(x, \xi)= \begin{cases}\frac{1}{W_{0}} \varphi_{1}(x) \varphi_{2}(\xi) & (x \leq \xi) \\ \frac{1}{W_{0}} \varphi_{1}(\xi) \varphi_{2}(x) & (x \geq \xi)\end{cases}
$$

where

$$
W_{0}=-p(a) W\left(\varphi_{1}, \varphi_{2}\right)(a)
$$


Observe that $W_{0} \neq 0$ since the existence of the Green function by Lemma 2.1 implies $B_{1}\left(\varphi_{2}\right) \neq 0$ and $B_{2}\left(\varphi_{1}\right) \neq 0$ and $\varphi_{1}, \varphi_{2}$ are linearly independent. We have

$$
p(x) \varphi_{1}^{\prime}(x)=p(a) \varphi_{1}^{\prime}(a)+\int_{a}^{x} r(t) \varphi_{1}(t) d t \geq \int_{a}^{x} r(t) \varphi_{1}(t) d t
$$

and

$$
p(x) \varphi_{2}^{\prime}(x)=p(b) \varphi_{2}^{\prime}(b)+\int_{b}^{x} r(t) \varphi_{2}(t) d t \leq \int_{b}^{x} r(t) \varphi_{2}(t) d t .
$$

It is now easy to see from $(2.2)$ and $(2.3)$ that $\varphi_{1}(x)>0, \varphi_{1}^{\prime}(x) \geq 0, \varphi_{2}(x)>0$ and $\varphi_{2}^{\prime}(x) \leq 0, a<x<b$. Hence, by noting $W_{0} \neq 0$, we obtain

$$
W_{0}=-p(a)\left(\varphi_{1}(a) \varphi_{2}^{\prime}(a)-\varphi_{2}(a) \varphi_{1}^{\prime}(a)\right)>0,
$$

and $G_{r}(x, \xi)>0$ for all $x, \xi \in(a, b)$.

Q.E.D.

LEMMA 2.4. We keep the notation and assumptions of Lemma 2.3. If $s(x) \in$ $C[a, b]$ and $r(x) \leq s(x), a \leq x \leq b$, then

$$
G_{r}(x, \xi) \geq G_{s}(x, \xi)
$$

for all $x, \xi \in[a, b]$.

Proof. Let $\mathscr{G}_{r}: C[a, b] \rightarrow \mathscr{D}$ be the Green operator defined by

$$
\mathscr{G}_{r} \varphi=\int_{a}^{b} G_{r}(x, \xi) \varphi(\xi) d \xi, \quad \varphi \in C[a, b] .
$$

If $\varphi \geq 0$, then, noting $\mathscr{G}_{r} \mathscr{L}_{r}=I=\mathscr{L}_{s} \mathscr{G}_{s}$, we have for any $x \in[a, b]$

$$
\begin{aligned}
\mathscr{G}_{r} \varphi-\mathscr{G}_{s} \varphi & =-\mathscr{G}_{r}\left(\mathscr{L}_{r}-\mathscr{L}_{s}\right) \mathscr{G}_{s} \varphi \\
& =-\mathscr{G}_{r}(r(x)-s(x)) \mathscr{G}_{s} \varphi \\
& =-\int_{a}^{b}\left[G_{r}(x, \xi)(r(\xi)-s(\xi)) \int_{a}^{b} G_{s}(\xi, \eta) \varphi(\eta) d \eta\right] d \xi \\
& =\int_{a}^{b}\left[G_{r}(x, \xi)(s(\xi)-r(\xi)) \int_{a}^{b} G_{s}(\xi, \eta) \varphi(\eta) d \eta\right] d \xi \\
& \geq 0
\end{aligned}
$$

where we have used Lemma 2.3 and the assumption $s(x) \geq r(x)$ in $[a, b]$. Hence

$$
\int_{a}^{b}\left\{G_{r}(x, \xi)-G_{s}(x, \xi)\right\} \varphi(\xi) \geq 0
$$

for any nonnegative function $\varphi \in C[a, b]$. This implies $G_{r}(x, \xi)-G_{s}(x, \xi) \geq 0$ for all $x, \xi \in[a, b]$.

Q.E.D. 
We are now in a position to prove the existence and uniqueness of solution for the problem (1.1)-(1.3).

THEOREM 2.1. Assume that $p \in C^{1}[a, b]$, and $f(x, u, v)$ is continuous on $\mathscr{R}=[a, b] \times \mathbb{R}^{2}$ and has continuous derivatives $\frac{\partial f}{\partial u}$ and $\frac{\partial f}{\partial v}$ there which satisfy, for some constants $K$ and $M$,

$$
0 \leq \frac{\partial f}{\partial u} \leq K
$$

and

$$
\left|\frac{\partial f}{\partial v}\right| \leq M .
$$

Then the equation (1.1) has a unique solution in $\mathscr{D}$ so that the boundary value problem (1.1)-(1.3) has a unique solution $u \in C^{2}[a, b]$.

Proof. For the sake of simplicity, we give a proof for the case $\alpha_{0} \alpha_{1} \beta_{0} \beta_{1} \neq 0$. The treatment of the other case is similar.

(i) Existence of Solution. We prove the existence of solution with the use of the Schauder fixed point theorem. Let $X=C^{1}[a, b]$ and define a norm $\|\cdot\|$ on $X$ by

$$
\|u\| \equiv\|u\|_{\infty}+\left\|u^{\prime}\right\|_{\infty}=\max _{a \leq x \leq b}|u(x)|+\max _{a \leq x \leq b}\left|u^{\prime}(x)\right|, u \in X .
$$

Then $(X,\|\cdot\|)$ is a Banach space. For each $u \in X$, we put

$$
\begin{aligned}
q(x ; u) & =\int_{0}^{1} \frac{\partial f}{\partial v}\left(x, \theta u(x), \theta u^{\prime}(x)\right) d \theta \\
r(x ; u) & =\int_{0}^{1} \frac{\partial f}{\partial u}\left(x, \theta u(x), \theta u^{\prime}(x)\right) d \theta \\
f_{0}(x) & =f(x, 0,0) .
\end{aligned}
$$

Then

$$
\begin{gathered}
f\left(x, u, u^{\prime}\right)=f_{0}(x)+q(x ; u) u^{\prime}+r(x ; u) u, \\
|q(x ; u)| \leq M, \quad r(x ; u) \geq 0
\end{gathered}
$$

and, by Lemma 2.1 , the linear boundary value problem

$$
\begin{gathered}
-\frac{d}{d x}\left(p(x) \frac{d w}{d x}\right)+q(x ; u) \frac{d w}{d x}+r(x ; u) w=-f_{0}(x), \quad a \leq x \leq b, \\
w \in \mathscr{D}
\end{gathered}
$$

has a unique solution $w=w(x ; u) \in C^{2}[a, b]$. 
To estimate $\|w\|$, we set

$$
\begin{gathered}
P(x ; u)=e^{\int_{a}^{x} \frac{p^{\prime}(t)-q(t ; u)}{p(t)} d t}, \quad R(x ; u)=\frac{P(x ; u)}{p(x)} r(x ; u), \quad g(x ; u)=-\frac{P(x ; u)}{p(x)} f_{0}(x), \\
P_{*}=\min _{a \leq x \leq b} e^{\int_{a}^{x} \frac{p^{\prime}(t)-M}{p(t)} d t}, \quad P^{*}=\max _{a \leq x \leq b} e^{\int_{a}^{x} \frac{p^{\prime}(t)+M}{p(t)} d t} .
\end{gathered}
$$

Then

$$
-\frac{d}{d x}\left(P(x ; u) \frac{d w}{d x}\right)+R(x ; u) w=g(x ; u), \quad P(x ; u)>0, R(x ; u) \geq 0
$$

so that

$$
w(x ; u)=\int_{a}^{b} \widehat{G}_{R}(x, \xi) g(\xi ; u(\xi)) d \xi,
$$

where $\widehat{G}_{R}(x, \xi)$ denotes the Green function for $\left(-\frac{d}{d x}\left(P(x ; u) \frac{d}{d x}[]\right)+R(x ; u)[], \mathscr{D}\right)$, whose existence is guaranteed by Lemma 2.1 .

In the following, for the sake of simplicity, we write $P(x ; u), R(x ; u), g(x ; u)$ and $w(x ; u)$ as $P(x), R(x), g(x)$ and $w(x)$, respectively. Then, by Lemmas $2.2-2.4$, we have

$$
0<\widehat{G}_{R}(x, \xi) \leq \widehat{G}_{0}(x, \xi) \equiv \widehat{G}(x, \xi) \leq \widehat{G}(x, x), \quad x, \xi \in[a, b]
$$

and

$$
\begin{aligned}
|w(x)| & \leq \int_{a}^{b} \widehat{G}(x, \xi)|g(\xi)| d \xi \\
& \leq \widehat{G}(x, x) \frac{P^{*}}{p_{*}}\left\|f_{0}\right\|_{\infty}(b-a),
\end{aligned}
$$

where $p_{*}=\min _{a \leq x \leq b} p(x)>0$. Furthermore, we have from Lemma 2.2

$$
\begin{aligned}
\widehat{G}(x, x)= & \frac{1}{P(a) P(b) \Delta}\left(\alpha_{1}+\alpha_{0} P(a) \int_{a}^{x} \frac{d t}{P(t)}\right) \\
& \times\left(\beta_{1}+\beta_{0} P(b) \int_{x}^{b} \frac{d t}{P(t)}\right) \\
\leq & \frac{1}{P_{*} \Delta}\left(\alpha_{1}+\alpha_{0} \int_{a}^{x} \frac{d t}{P_{*}}\right)\left(\beta_{1}+\beta_{0} P^{*} \int_{x}^{b} \frac{d t}{P_{*}}\right) \\
\leq & \frac{1}{P_{*} \Delta}\left(\alpha_{1}+\alpha_{0} \frac{b-a}{P_{*}}\right)\left(\beta_{1}+\beta_{0} \frac{P^{*}}{P_{*}}(b-a)\right)=\frac{1}{P_{*} \Delta} \gamma
\end{aligned}
$$

where

$$
\begin{aligned}
\Delta & =\alpha_{0}\left(\beta_{0} \int_{a}^{b} \frac{d t}{P(t)}+\frac{\beta_{1}}{P(b)}\right)+\frac{\alpha_{1} \beta_{0}}{P(a)} \\
& =\alpha_{0}\left(\beta_{0} \int_{a}^{b} \frac{d t}{P(t)}+\frac{\beta_{1}}{P(b)}\right)+\alpha_{1} \beta_{0} \\
& \geq \Delta_{*} \equiv \frac{\alpha_{0}}{P^{*}}\left(\beta_{0}(b-a)+\beta_{1}\right)+\alpha_{1} \beta_{0}>0
\end{aligned}
$$


and

$$
\gamma=\left(\alpha_{1}+\alpha_{0} \frac{b-a}{P_{*}}\right)\left(\beta_{1}+\beta_{0} \frac{P^{*}}{P_{*}}(b-a)\right) .
$$

Hence

$$
|w(x)| \leq \frac{1}{P_{*} \Delta_{*}} \gamma \frac{P^{*}}{p_{*}}\left\|f_{0}\right\|_{\infty}(b-a) \equiv \delta_{0} \quad(\text { say }) .
$$

On the other hand, it follows from

$$
-\frac{d}{d x}\left(P(x) \frac{d w}{d x}\right)=g(x)-R(x) w, \quad w \in \mathscr{D}
$$

that

$$
w(x)=\int_{a}^{b} \widehat{G}(x, \xi)(g(\xi)-R(\xi) w(\xi)) d \xi
$$

and an application of Lemma 2.2 yields

$$
\begin{aligned}
\frac{d w(x)}{d x}= & \frac{\partial}{\partial x}\left(\int_{a}^{x}+\int_{x}^{b}\right) \widehat{G}(x, \xi)(g(\xi)-R(\xi) w(\xi)) d \xi \\
= & \widehat{G}(x, x-0)(g(x)-R(x) w(x))-\widehat{G}(x, x+0)(g(x)-R(x) w(x)) \\
& +\left(\int_{a}^{x}+\int_{x}^{b}\right) \frac{\partial \widehat{G}(x, \xi)}{\partial x}(g(\xi)-R(\xi) w(\xi)) d \xi \\
= & \int_{a}^{x} \frac{-\beta_{0}}{\Delta P(x)}\left(\alpha_{1}+\alpha_{0} \int_{a}^{\xi} \frac{d t}{P(t)}\right)(g(\xi)-R(\xi) w(\xi)) d \xi \\
& +\int_{x}^{b} \frac{\alpha_{0}}{P(b) \Delta P(x)}\left(\beta_{1}+\beta_{0} P(b) \int_{\xi}^{b} \frac{d t}{P(t)}\right)(g(\xi)-R(\xi) w(\xi)) d \xi
\end{aligned}
$$

where $\Delta$ is defined by (2.6). Since

$$
\begin{aligned}
\Delta & =\beta_{0}\left(\alpha_{1}+\alpha_{0} \int_{a}^{b} \frac{d t}{P(t)}\right)+\frac{\alpha_{0} \beta_{1}}{P(b)} \\
& \geq \beta_{0}\left(\alpha_{1}+\alpha_{0} \int_{a}^{\xi} \frac{d t}{P(t)}\right) \quad \forall \xi \in[a, b]
\end{aligned}
$$

and

$$
\begin{aligned}
P(b) \Delta & =\alpha_{0}\left(\beta_{0} P(b) \int_{a}^{b} \frac{d t}{P(t)}+\beta_{1}\right)+\alpha_{1} \beta_{0} P(b) \\
& \geq \alpha_{0}\left(\beta_{0} P(b) \int_{\xi}^{b} \frac{d t}{P(t)}+\beta_{1}\right) \quad \forall \xi \in[a, b]
\end{aligned}
$$


we obtain

$$
\begin{aligned}
\left|\frac{d w(x)}{d x}\right| & \leq \int_{a}^{x} \frac{1}{P(x)}|g(\xi)-R(\xi) w(\xi)| d \xi+\int_{x}^{b} \frac{1}{P(x)}|g(\xi)-R(\xi) w(\xi)| d \xi \\
& =\int_{a}^{b} \frac{1}{P(x)}|g(\xi)-R(\xi) w(\xi)| d \xi \\
& \leq \frac{1}{P_{*}}\left(\|g\|_{\infty}+\|R\|_{\infty}\|w\|_{\infty}\right)(b-a) \\
& \leq \frac{1}{P_{*}}\left(\frac{P^{*}}{p_{*}}\left\|f_{0}\right\|_{\infty}+\frac{P^{*}}{p_{*}}\|r\|_{\infty} \delta_{0}\right)(b-a) \\
& \leq \frac{P^{*}}{P_{*} p_{*}}\left(\left\|f_{0}\right\|_{\infty}+K \delta_{0}\right)(b-a) \equiv \delta_{1} \quad \text { (say). }
\end{aligned}
$$

Hence, putting $\delta=\delta_{0}+\delta_{1}$ and

$$
\mathscr{S}=\left\{u \in X \mid\|u\| \leq \delta, B_{1}(u)=B_{2}(u)=0\right\}
$$

we have

$$
\|w\|=\|w\|_{\infty}+\left\|w^{\prime}\right\|_{\infty} \leq \delta_{0}+\delta_{1}=\delta \quad \forall u \in \mathscr{S} .
$$

A continuous map $T: \mathscr{S} \rightarrow C^{2}[a, b] \cap \mathscr{S} \subset \mathscr{S}$ is thus defined by $T u=w$ for $u \in \mathscr{S}$. $\mathscr{S}$ is a closed convex subset of $X$ and the set $\mathscr{F}=T(\mathscr{S})$ is equicontinuous, since

$$
\left|w\left(t_{1}\right)-w\left(t_{2}\right)\right| \leq\left|\int_{t_{2}}^{t_{1}}\right| w^{\prime}(t)|d t| \leq \delta_{1}\left|t_{1}-t_{2}\right|, \quad t_{1}, t_{2} \in[a, b] .
$$

Hence, by Ascoli-Arzela's theorem, any infinite sequence $\left\{w_{n}\right\}, n=1,2, \cdots$ of $\mathscr{F}$ contains a subsequence $\left\{w_{n_{j}}\right\}, n_{1}<n_{2}<\cdots$ which converges uniformly on $[a, b]$ to a function $\widetilde{w} \in C[a, b]$ as $j \rightarrow \infty$. Then the set $\mathscr{F}^{\prime}=\left\{w_{n_{j}}^{\prime}\right\}$ is also uniformly bounded and equicontinuous since $\left\|w_{n_{j}}^{\prime}\right\|_{\infty} \leq \delta_{1} \quad \forall j$ and

$$
\begin{aligned}
w_{n_{j}}^{\prime}\left(t_{1}\right)-w_{n_{j}}^{\prime}\left(t_{2}\right) & =\int_{t_{2}}^{t_{1}} w_{n_{j}}^{\prime \prime}(t) d t \\
& =\int_{t_{2}}^{t_{1}} \frac{1}{p}\left[-p^{\prime} w_{n_{j}}^{\prime}+q w_{n_{j}}^{\prime}+r w_{n_{j}}+f_{0}\right] d t, \\
& t_{1}, t_{2} \in[a, b]
\end{aligned}
$$

so that

$$
\left|w_{n_{j}}^{\prime}\left(t_{1}\right)-w_{n_{j}}^{\prime}\left(t_{2}\right)\right| \leq \frac{1}{p_{*}}\left\{\left(\left\|p^{\prime}\right\|_{\infty}+M\right) \delta_{1}+K \delta_{0}+\left\|f_{0}\right\|_{\infty}\right\}\left|t_{1}-t_{2}\right| .
$$

Therefore, we can again employ the Ascoli-Arzela theorem to extract a uniformly convergent subsequence $\left\{w_{n_{j_{k}}}^{\prime}\right\}$ of $\mathscr{F}^{\prime}$. Then it is easy to see that the limit function 
$\widetilde{w}$ of $\left\{w_{n_{j}}\right\}$ is differentiable and $\widetilde{w}^{\prime}$ is the limit function of $\left\{w_{n_{j_{k}}}^{\prime}\right\}: w_{n_{j_{k}}}^{\prime} \rightarrow \widetilde{w}^{\prime}$ as $k \rightarrow \infty$ with respect to the norm $\|\cdot\|_{\infty}$. Hence

$$
\left\|w_{n_{j_{k}}}-\widetilde{w}\right\|=\left\|w_{n_{j_{k}}}-\widetilde{w}\right\|_{\infty}+\left\|w_{n_{j_{k}}}^{\prime}-\widetilde{w}^{\prime}\right\|_{\infty} \rightarrow 0
$$

as $k \rightarrow \infty$, which implies that the set $\mathscr{F}$ is relatively compact in the Banach space $(X,\|\cdot\|)$. Consequently we can apply the Schauder fixed point theorem to conclude that $T$ has a fixed point $u$ in $\mathscr{S}$. Since $T u \in C^{2}[a, b]$ by definition of $T$, we obtain $u(=T u) \in C^{2}[a, b]$ and $u$ is a solution of (1.1) in $\mathscr{D}$, which proves the existence of a solution for (1.1) in $\mathscr{D}$. Hence the problem (1.1)-(1.3) has a solution in $C^{2}[a, b]$.

(ii) Uniqueness. Let $u$ and $v$ be two solutions for (1.1)-(1.3), which belong to $C^{2}[a, b]$, and put $w=u-v$. Then

$$
f\left(x, u, u^{\prime}\right)-f\left(x, v, v^{\prime}\right)=r(x) w+q(x) w^{\prime}
$$

with

$$
\begin{aligned}
& r(x)=r(x ; u, v):=\int_{0}^{1} f_{u}\left(x, v+\theta w, v^{\prime}+\theta w^{\prime}\right) d \theta \\
& q(x)=q(x ; u, v):=\int_{0}^{1} f_{u^{\prime}}\left(x, v+\theta w, v^{\prime}+\theta w^{\prime}\right) d \theta
\end{aligned}
$$

which leads to the equation

$$
-\left(p w^{\prime}\right)^{\prime}+q w^{\prime}+r w=0, \quad a \leq x \leq b, \quad w \in \mathscr{D} .
$$

We again put

$$
P(x)=e^{\int_{a}^{x} \frac{p^{\prime}(t)-q(t)}{p(t)} d t}, \quad R(x)=P(x) \frac{r(x)}{p(x)}
$$

and rewrite $(2.9)$ as

$$
-\frac{d}{d x}\left(P(x) \frac{d w}{d x}\right)+R(x) w=0, \quad w \in \mathscr{D} .
$$

Multiplying both sides by $w(x)$ and integrating from $a$ to $b$, we have

$$
\left[-P(x) \frac{d w}{d x} w\right]_{a}^{b}+\int_{a}^{b}\left\{P(x)\left(\frac{d w}{d x}\right)^{2}+R(x) w^{2}\right\} d x=0 .
$$

As in the proof of Lemma 2.1, we can show

$$
\left[-P(x) \frac{d w}{d x} w\right]_{a}^{b} \geq 0
$$

and $w \equiv 0$ since $\alpha_{0}+\beta_{0}>0$ by (1.7). This proves the uniqueness of solution for (1.1)-(1.3), which completes the proof of Theorem 2.1.

Q.E.D. 
REMARK 2.1. If $f=f(x, u)$, then the conditions (2.4) in Theorem 2.1 can be replaced by weaker condition $\frac{\partial f}{\partial u} \geq 0$. In fact, for such a function $f$, the above proof shows that we can replace the constant $K$ in (2.8) and the set $\mathscr{S}$ by

$$
\sup _{[a, b] \times\left[-\delta_{0}, \delta_{0}\right]} f_{u}(x, u)
$$

and

$$
\widetilde{\mathscr{S}}=\left\{u \in X \mid\|u\| \leq \delta,\|u\|_{\infty} \leq \delta_{0}, B_{1}(u)=B_{2}(u)=0\right\}
$$

respectively, and the similar argument can be applied to prove the existence of a solution of (1.1) in $\mathscr{D}$. This improves and generalizes Henrici's result mentioned in $\S 1$.

\section{A Discretization for (1.1)-(1.3)}

To discretize (1.1)-(1.3) under the assumptions of Theorem 2.1, we consider the case $\alpha_{0} \alpha_{1} \beta_{0} \beta_{1} \neq 0$ for the sake of simplicity. The treatment of other cases is similar. We take any nodes $\left\{x_{i}\right\}$ as in (1.4) and set

$$
\begin{aligned}
& x_{i+\frac{1}{2}}=\frac{1}{2}\left(x_{i}+x_{i+1}\right) \\
& \omega_{i}= \begin{cases}\frac{1}{2} h_{1} & (i=0) \\
\frac{1}{2}\left(h_{i}+h_{i+1}\right) & (1 \leq i \leq n) \\
\frac{1}{2} h_{n+1} & (i=n+1)\end{cases} \\
& H=\operatorname{diag}\left(\frac{1}{\omega_{0}}, \frac{1}{\omega_{1}}, \ldots, \frac{1}{\omega_{n+1}}\right) \\
& a_{i}= \begin{cases}\frac{\alpha_{0}}{\alpha_{1}} p(a) & (i=0) \\
\frac{1}{\int_{x_{i-1}}^{x_{i}} \frac{d t}{p(t)}} & (1 \leq i \leq n+1) \\
\frac{\beta_{0}}{\beta_{1}} p(b) & (i=n+2)\end{cases} \\
& A=\left(\begin{array}{cccccc}
a_{0}+a_{1} & -a_{1} & & & & \\
-a_{1} & a_{1}+a_{2} & -a_{2} & & & \\
\ddots & \ddots & \ddots & & & \\
& & & -a_{n} & a_{n}+a_{n+1} & -a_{n+1} \\
& & & & -a_{n+1} & a_{n+1}+a_{n+2}
\end{array}\right)
\end{aligned}
$$




$$
\boldsymbol{f}(\boldsymbol{U})=\left(\begin{array}{c}
f\left(x_{0}, U_{0}, \frac{\alpha_{0} U_{0}-\alpha}{\alpha_{1}}\right) \\
f\left(x_{1}, U_{1}, \frac{U_{2}-U_{0}}{h_{1}+h_{2}}\right) \\
\vdots \\
f\left(x_{n}, U_{n}, \frac{U_{n+1}-U_{n-1}}{h_{n}+h_{n+1}}\right) \\
f\left(x_{n+1}, U_{n+1}, \frac{\beta-\beta_{0} U_{n+1}}{\beta_{1}}\right)
\end{array}\right),
$$

Then a discretized system which we propose here is

$$
H A \boldsymbol{U}+\widetilde{\boldsymbol{f}}(\boldsymbol{U})=0 .
$$

The matrix $A$ in (3.4) has the following property.

Lemma 3.1 ([16]). Let $\mathscr{L}$ be the operator defined as in Lemma 2.2 and $G(x, \xi)$ be the Green function for $(\mathscr{L}, \mathscr{D})$. Then $A^{-1}=\left(G\left(x_{i}, x_{j}\right)\right)$.

Proof. Let $A^{-1}=\left(\alpha_{i j}\right)$. Then by an inversion formula for tridiagonal matrices [14], we have

$$
\alpha_{i j}= \begin{cases}\frac{1}{z_{n+2}} z_{i}\left(z_{n+2}-z_{j}\right) & (i \leq j) \\ \frac{1}{z_{n+2}} z_{j}\left(z_{n+2}-z_{i}\right) & (i \geq j),\end{cases}
$$

where

$$
z_{i}=\sum_{k=0}^{i} \frac{1}{a_{k}}= \begin{cases}\frac{\alpha_{1}}{\alpha_{0} p(a)} & (i=0) \\ \frac{\alpha_{1}}{\alpha_{0} p(a)}+\int_{a}^{x_{i}} \frac{d t}{p(t)} & (1 \leq i \leq n+1) \\ \frac{\alpha_{1}}{\alpha_{0} p(a)}+\frac{\beta_{1}}{\beta_{0} p(b)}+\int_{a}^{b} \frac{d t}{p(t)} & (i=n+2) .\end{cases}
$$

Therefore

$$
z_{n+2}-z_{j}=\sum_{k=j+1}^{n+2} \frac{1}{a_{k}}=\frac{\beta_{1}}{\beta_{0} p(b)}+\int_{x_{j}}^{b} \frac{d t}{p(t)} .
$$


Comparing (3.5)-(3.7) with the expression of $G(x, \xi)$ in Lemma 2.2, we see $\alpha_{i j}=$ $G\left(x_{i}, x_{j}\right)$.

Q.E.D.

\section{Existence of Solution for (3.4)}

Since the discretized system (3.4) is nonlinear if $f$ is nonlinear, it is interesting to know whether (3.4) has a unique solution or not. In this section we shall show that, under weaker assumptions than those of Theorem 2.1 (that is, (2.4) is replaced by $\left.f_{u} \geq 0\right)$, (3.4) has a unique solution if $h=\max _{i} h_{i}$ is sufficiently small. This is done along the similar line as in $\S 2$.

Let

$$
\begin{aligned}
& d_{0}=\int_{0}^{1} f_{u}\left(x_{0}, \theta U_{0}, \frac{-\alpha+\theta \alpha_{0} U_{0}}{\alpha_{1}}\right) d \theta \\
& d_{i}=\int_{0}^{1} f_{u}\left(x_{i}, \theta U_{i}, \theta \frac{U_{i+1}-U_{i-1}}{h_{i+1}+h_{i}}\right) d \theta \quad(1 \leq i \leq n) \\
& d_{n+1}=\int_{0}^{1} f_{u}\left(x_{n+1}, \theta U_{n+1}, \frac{\beta-\theta \beta_{0} U_{n+1}}{\beta_{1}}\right) d \theta \\
& \sigma_{0}=\frac{\alpha_{0}}{\alpha_{1}} \omega_{0} \int_{0}^{1} f_{u^{\prime}}\left(x_{0}, \theta U_{0}, \frac{-\alpha+\theta \alpha_{0} U_{0}}{\alpha_{1}}\right) d \theta \\
& \sigma_{i}=\frac{1}{2} \int_{0}^{1} f_{u^{\prime}}\left(x_{i}, \theta U_{i}, \theta \frac{U_{i+1}-U_{i-1}}{h_{i+1}+h_{i}}\right) d \theta \quad(1 \leq i \leq n) \\
& \sigma_{n+1}=-\frac{\beta_{0}}{\beta_{1}} \omega_{n+1} \int_{0}^{1} f_{u^{\prime}}\left(x_{n+1}, \theta U_{n+1}, \frac{\beta-\theta \beta_{0} U_{n+1}}{\beta_{1}}\right) d \theta \\
& D=\operatorname{diag}\left(d_{0}, d_{1}, \ldots, d_{n+1}\right)
\end{aligned}
$$

and

$$
E=\left(\begin{array}{ccccc}
\sigma_{0} & 0 & & & \\
-\sigma_{1} & 0 & \sigma_{1} & & \\
& \ddots & \ddots & \ddots & \\
& & -\sigma_{n} & 0 & \sigma_{n} \\
& & & 0 & \sigma_{n+1}
\end{array}\right)
$$

Then (3.4) can be written

$$
H\left(A+E+H^{-1} D\right) \boldsymbol{U}=-\tilde{\boldsymbol{f}}(\mathbf{0})
$$

since

$$
\widetilde{\boldsymbol{f}}(\boldsymbol{U})=\widetilde{\boldsymbol{f}}(\mathbf{0})+\left(\int_{0}^{1} \widetilde{\boldsymbol{f}}^{\prime}(\theta \boldsymbol{U}) d \theta\right) \boldsymbol{U}
$$


where

$$
\widetilde{\boldsymbol{f}}(\mathbf{0})=\left(\begin{array}{c}
f\left(x_{0}, 0,-\frac{\alpha}{\alpha_{1}}\right)-\frac{2}{h_{1}} \frac{\alpha}{\alpha_{1}} p_{0} \\
f\left(x_{1}, 0,0\right) \\
\vdots \\
f\left(x_{n}, 0,0\right) \\
f\left(x_{n+1}, 0, \frac{\beta}{\beta_{1}}\right)-\frac{2}{h_{n+1}} \frac{\beta}{\beta_{1}} p_{n+1}
\end{array}\right) .
$$

Furthermore, $Z=A+E+H^{-1} D$ is an irreducibly diagonally dominant $L$ matrix so that $Z$ is nonsingular and, given $\boldsymbol{U} \in \mathbb{R}^{n+2}$, the linear system

$$
H Z \boldsymbol{W}=-\widetilde{\boldsymbol{f}}(\mathbf{0})
$$

has a unique solution $\boldsymbol{W}=-Z^{-1} H^{-1} \tilde{\boldsymbol{f}}(\mathbf{0})$. In order to estimate $\|\boldsymbol{W}\|_{\infty}$, we take a diagonal matrix $Q=\operatorname{diag}\left(1, \rho_{1}, \ldots, \rho_{n+1}\right)$ with $\rho_{i}>0$ such that $B=Q(A+E)$ is symmetric. We then have

$$
B=\left(\begin{array}{cccc}
\widehat{a}_{0}+a_{1} & -a_{1} & & \\
-\rho_{1}\left(a_{1}+\sigma_{1}\right) & \rho_{1}\left(a_{1}+a_{2}\right) & -\rho_{1}\left(a_{2}-\sigma_{1}\right) & \\
\ddots & \ddots & \ddots & \\
& -\rho_{n}\left(a_{n}+\sigma_{n}\right) & \rho_{n}\left(a_{n}+a_{n+1}\right) & -\rho_{n}\left(a_{n+1}-\sigma_{n}\right) \\
& & -\rho_{n+1} a_{n+1} & \rho_{n+1} a_{n+1}+\rho_{n+1} \widehat{a}_{n+2}
\end{array}\right)
$$

where

$$
\begin{aligned}
& \widehat{a}_{0}=a_{0}+\sigma_{0}=a_{0}+O\left(h_{1}\right), \\
& \widehat{a}_{n+2}=a_{n+2}+\sigma_{n+1}=a_{n+2}+O\left(h_{n+1}\right) .
\end{aligned}
$$

LEMMA 4.1. If $h$ is sufficiently so small that $M \int_{x_{i-1}}^{x_{i}} \frac{d t}{p(t)} \leq 1 \forall i$, then

$$
e^{-\frac{3 M}{2} \int_{a}^{x_{i}} \frac{d t}{p(t)}} \leq \rho_{i} \leq e^{\frac{3 M}{2} \int_{a}^{x_{i}} \frac{d t}{p(t)}}, \quad 1 \leq i \leq n+1
$$

Proof. Since $B$ is symmetric, we have

$$
\rho_{1}=\frac{a_{1}}{a_{1}+\sigma_{1}}, \quad \rho_{i}=\frac{a_{i}-\sigma_{i-1}}{a_{i}+\sigma_{i}} \rho_{i-1}(2 \leq i \leq n), \quad \rho_{n+1}=\frac{a_{n+1}-\sigma_{n}}{a_{n+1}} \rho_{n} .
$$

We remark here that the assumptions $M \int_{x_{i-1}}^{x_{i}} \frac{d t}{p(t)} \leq 1 \quad \forall i$ imply $\rho_{i}>0$, $1 \leq i \leq n+1$. In fact, we have

$$
\left|\sigma_{i}\right| \leq \frac{M}{2}, \quad 1 \leq i \leq n
$$


so that

$$
a_{i}+\sigma_{i} \geq \frac{1}{\int_{x_{i-1}}^{x_{i}} \frac{d t}{p(t)}}-\frac{M}{2}>0, \quad 1 \leq i \leq n
$$

and

$$
a_{i}-\sigma_{i-1} \geq \frac{1}{\int_{x_{i-1}}^{x_{i}} \frac{d t}{p(t)}}-\frac{M}{2}>0, \quad 2 \leq i \leq n+1 .
$$

In the following, we use elementary inequalities

$$
1-\theta \geq \frac{1}{1+2 \theta} \geq e^{-2 \theta} \quad \text { and } \quad e^{2 \theta} \geq \frac{1}{1-\theta} \geq e^{\theta}
$$

if $0 \leq \theta \leq \frac{1}{2}$. Then, we have

$$
0<\rho_{1}<\frac{1}{1-\frac{M}{2 a_{1}}} \leq e^{\frac{M}{a_{1}}}<e^{\frac{3 M}{2 a_{1}}}
$$

and, by induction on $i(2 \leq i \leq n)$

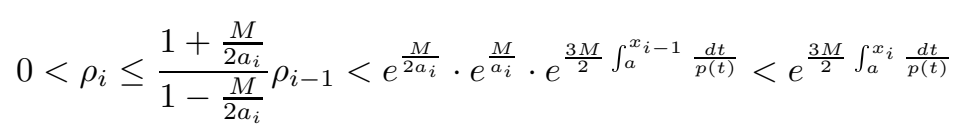

Furthermore, we have

$$
\begin{aligned}
0<\rho_{n+1} & =\left(1-\frac{\sigma_{n}}{a_{n+1}}\right) \rho_{n} \leq\left(1+\frac{M}{2 a_{n+1}}\right) \rho_{n} \\
& <e^{\frac{M}{2 a_{n+1}}} \cdot e^{\frac{3 M}{2} \int_{a}^{x_{n}} \frac{d t}{p(t)}}<e^{\frac{3 M}{2} \int_{a}^{x_{n+1}} \frac{d t}{p(t)}}
\end{aligned}
$$

Similarly, we have

$$
\begin{aligned}
& \rho_{1} \geq \frac{1}{1+\frac{M}{2 a_{1}}} \geq \frac{1}{e^{\frac{M}{2 a_{1}}}} \geq e^{-\frac{3 M}{2} \int_{a}^{x_{1}} \frac{d t}{p(t)}} \\
& \rho_{i} \geq \frac{1-\frac{M}{2 a_{i}}}{1+\frac{M}{2 a_{i}}} \rho_{i-1} \geq \frac{e^{-\frac{M}{a_{i}}}}{e^{\frac{M}{2 a_{i}}}} \cdot e^{-\frac{3 M}{2} \int_{a}^{x_{i}-1}} \frac{d t}{p(t)}=e^{-\frac{3 M}{2} \int_{a}^{x_{i}} \frac{d t}{p(t)}} \quad(2 \leq i \leq n)
\end{aligned}
$$

and

$$
\rho_{n+1} \geq\left(1-\frac{M}{2 a_{n+1}}\right) \rho_{n}>e^{-\frac{M}{a_{n+1}}} \cdot e^{-\frac{3 M}{2} \int_{a}^{x_{n}} \frac{d t}{p(t)}}>e^{-\frac{3 M}{2} \int_{a}^{x_{n+1}} \frac{d t}{p(t)}}
$$

Q.E.D. 
Lemma 4.2. Let $B$ be the symmetric matrix defined as in (4.2). If $h$ is sufficiently small, then

$$
B^{-1} \leq e^{7 M \int_{a}^{b} \frac{d t}{p(t)}} \cdot A^{-1} .
$$

Proof. Let $B^{-1}=\left(\beta_{i j}\right)$. Then, as in the proof of Lemma 3.1, we have

$$
\beta_{i j}= \begin{cases}\frac{1}{z_{n+2}} z_{i}\left(z_{n+2}-z_{j}\right) & (i \leq j) \\ \frac{1}{z_{n+2}} z_{j}\left(z_{n+2}-z_{i}\right) & (i \geq j)\end{cases}
$$

where

$$
z_{i}= \begin{cases}\frac{1}{\widehat{a}_{0}} & (i=0) \\ z_{i-1}+\frac{1}{\rho_{i}\left(a_{i}+\sigma_{i}\right)} & (1 \leq i \leq n) \\ z_{i-1}+\frac{1}{\rho_{n+1} a_{n+1}} & (i=n+1) \\ z_{i-1}+\frac{1}{\rho_{n+1} \widehat{a}_{n+2}} & (i=n+2) .\end{cases}
$$

We repeat the same argument as in the proof of Lemma 4.1 to obtain

$$
z_{i}<\left(\frac{1}{a_{0}}+\cdots+\frac{1}{a_{i}}\right) e^{\frac{5 M}{2} \int_{a}^{b} \frac{d t}{p(t)}}, \quad 0 \leq i \leq n+1 .
$$

In fact, we have for sufficiently small $h$

$$
\begin{aligned}
& z_{0}=\frac{1}{\widehat{a}_{0}}=\frac{1}{a_{0}+\sigma_{0}} \leq \frac{1}{a_{0}\left(1-\frac{M h_{1}}{2 p(a)}\right)} \leq \frac{1}{a_{0}} e^{\frac{M h_{1}}{p(a)}}<\frac{1}{a_{0}} e^{\frac{5 M}{2} \int_{a}^{b} \frac{d t}{p(t)}} \\
& z_{1}=z_{0}+\frac{1}{a_{1}}<\left(\frac{1}{a_{0}}+\frac{1}{a_{1}}\right) e^{\frac{5 M}{2} \int_{a}^{b} \frac{d t}{p(t)}}
\end{aligned}
$$

By induction on $i(2 \leq i \leq n)$

$$
\begin{aligned}
z_{i} & =z_{i-1}+\frac{1}{\rho_{i}\left(a_{i}+\sigma_{i}\right)} \leq z_{i-1}+\frac{1}{a_{i}} \cdot e^{\frac{3 M}{2} \int_{a}^{x_{i}} \frac{d t}{p(t)}} \cdot e^{M \int_{x_{i-1}}^{x_{i}} \frac{d t}{p(t)}} \\
& \leq\left(\frac{1}{a_{0}}+\cdots+\frac{1}{a_{i-1}}\right) e^{\frac{5 M}{2} \int_{a}^{b} \frac{d t}{p(t)}}+\frac{1}{a_{i}} e^{\frac{5 M}{2} \int_{a}^{x_{i}} \frac{d t}{p(t)}} \\
& <\left(\frac{1}{a_{0}}+\cdots+\frac{1}{a_{i}}\right) e^{\frac{5 M}{2} \int_{a}^{b} \frac{d t}{p(t)}}
\end{aligned}
$$

Furthermore, we have

$$
\begin{aligned}
z_{n+1} & =z_{n}+\frac{1}{\rho_{n+1} a_{n+1}} \leq\left(\frac{1}{a_{0}}+\cdots+\frac{1}{a_{n}}\right) e^{\frac{5 M}{2} \int_{a}^{b} \frac{d t}{p(t)}}+\frac{1}{a_{n+1}} e^{\frac{3 M}{2} \int_{a}^{b} \frac{d t}{p(t)}} \\
& <\left(\frac{1}{a_{0}}+\cdots+\frac{1}{a_{n+1}}\right) e^{\frac{5 M}{2} \int_{a}^{b} \frac{d t}{p(t)}}
\end{aligned}
$$


On the other hand,

$$
z_{n+2}-z_{j}= \begin{cases}\sum_{k=j+1}^{n} \frac{1}{\rho_{k}\left(a_{k}+\sigma_{k}\right)}+\frac{1}{\rho_{n+1} a_{n+1}}+\frac{1}{\rho_{n+1} \widehat{a}_{n+2}} & (0 \leq j \leq n-1) \\ \frac{1}{\rho_{n+1} a_{n+1}}+\frac{1}{\rho_{n+1} \widehat{a}_{n+2}} & (j=n) \\ \frac{1}{\rho_{n+1} \widehat{a}_{n+2}} & (j=n+1) .\end{cases}
$$

Hence, if $0 \leq j \leq n-1$, then

$$
\begin{aligned}
z_{n+2}-z_{j} \leq & \sum_{k=j+1}^{n} \frac{1}{a_{k}} \cdot e^{\frac{3 M}{2} \int_{a}^{x_{k}} \frac{d t}{p(t)}} \cdot e^{M \int_{x_{k-1}}^{x_{k}} \frac{d t}{p(t)}}+\frac{1}{a_{n+1}} \cdot e^{\frac{3 M}{2} \int_{a}^{x_{n+1}} \frac{d t}{p(t)}} \\
& +\frac{1}{a_{n+2}} \cdot e^{\frac{3 M}{2} \int_{a}^{x_{n+1}} \frac{d t}{p(t)}} \cdot e^{M \int_{x_{n}}^{x_{n}+1} \frac{d t}{p(t)}} \\
< & \left(\sum_{k=j+1}^{n+2} \frac{1}{a_{k}}\right) e^{\frac{5 M}{2} \int_{a}^{b} \frac{d t}{p(t)}}
\end{aligned}
$$

Similarly, we have

$$
\begin{aligned}
z_{n+2}-z_{n} & =\frac{1}{\rho_{n+1} a_{n+1}}+\frac{1}{\rho_{n+1} \widehat{a}_{n+2}}<\left(\frac{1}{a_{n+1}}+\frac{1}{a_{n+2}}\right) e^{\frac{5 M}{2} \int_{a}^{b} \frac{d t}{p(t)}}, \\
z_{n+2}-z_{n+1} & <\frac{1}{a_{n+2}} e^{\frac{5 M}{2} \int_{a}^{b} \frac{d t}{p(t)}}
\end{aligned}
$$

and

$$
z_{n+2}>\left(\frac{1}{a_{0}}+\cdots+\frac{1}{a_{n+2}}\right) e^{-2 M \int_{a}^{b} \frac{d t}{p(t)}},
$$

since

$$
\begin{array}{r}
\frac{1}{\rho_{i}\left(a_{i}+\sigma_{i}\right)}>\frac{1}{a_{i}} e^{-\frac{3 M}{2} \int_{a}^{x_{i}} \frac{d t}{p(t)}} \cdot e^{-\frac{M}{2} \int_{x_{i-1}}^{x_{i}} \frac{d t}{p(t)}} \geq \frac{1}{a_{i}} e^{-2 M \int_{a}^{x_{i}} \frac{d t}{p(t)}}, \\
1 \leq i \leq n, \text { etc. }
\end{array}
$$

Consequently, we obtain

$$
\begin{aligned}
\beta_{i j} \leq & \frac{\left(\frac{1}{a_{0}}+\cdots+\frac{1}{a_{i}}\right) \cdot e^{\frac{5 M}{2} \int_{a}^{b} \frac{d t}{p(t)}} \cdot\left(\sum_{k=j+1}^{n+2} \frac{1}{a_{k}}\right) \cdot e^{\frac{5 M}{2} \int_{a}^{b} \frac{d t}{p(t)}}}{\left(\frac{1}{a_{0}}+\cdots+\frac{1}{a_{n+2}}\right) \cdot e^{-2 M \int_{a}^{b} \frac{d t}{p(t)}}} \\
= & \alpha_{i j} \cdot e^{7 M \int_{a}^{b} \frac{d t}{p(t)}} \\
= & G\left(x_{i}, x_{j}\right) \cdot e^{7 M \int_{a}^{b} \frac{d t}{p(t)}} .
\end{aligned}
$$

Q.E.D. 
We are now in a position to prove the following theorem:

Theorem 4.1. Let $p \in C^{1}[a, b]$ and $f(x, u, v)$ be continuous on $\mathscr{R}=[a, b] \times$ $\mathbb{R} \times \mathbb{R}$ and have continuous derivatives $f_{u}, f_{v}$ there which satisfy

$$
f_{u} \geq 0 \text { and }\left|f_{v}\right| \leq M
$$

where $M$ is a constant. If $h$ is sufficiently small, then the equation (3.4) has a unique solution. (Note that the conditions (2.4) in Theorem 2.1 are relaxed to $f_{u} \geq 0$.)

Proof. (i) Uniqueness. Let

$$
\boldsymbol{U}=\left(U_{0}, \ldots, U_{n+1}\right)^{t} \text { and } \boldsymbol{V}=\left(V_{0}, \ldots, V_{n+1}\right)^{t}
$$

be two solutions of (3.4) and put $\boldsymbol{W}=\boldsymbol{U}-\boldsymbol{V}=\left(W_{0}, \ldots, W_{n+1}\right)^{t}$. Then

$$
H A \boldsymbol{W}+\widetilde{\boldsymbol{f}}(\boldsymbol{U})-\tilde{\boldsymbol{f}}(\boldsymbol{V})=0 .
$$

We obtain from (4.3)

$$
(H A+J) \boldsymbol{W}=\mathbf{0},
$$

where

$$
J=\int_{0}^{1} \widetilde{\boldsymbol{f}}^{\prime}(\boldsymbol{V}+\theta(\boldsymbol{U}-\boldsymbol{V})) d \theta=\int_{0}^{1} \boldsymbol{f}^{\prime}(\boldsymbol{V}+\theta(\boldsymbol{U}-\boldsymbol{V})) d \theta .
$$

As is easily seen, $H A+J$ is an irreducibly diagonally dominant $L$-matrix so that $H A+J$ is nonsingular, which implies $\boldsymbol{W}=\mathbf{0}$.

(ii) Existence. We apply the Brouwer fixed point theorem to derive the existence of solution for (3.4). Given $\boldsymbol{U} \in \mathbb{R}^{n+2}$, consider the linear system (4.1). Then

$$
\begin{aligned}
|\boldsymbol{W}| & =\left|-Z^{-1} H^{-1} \tilde{\boldsymbol{f}}(\mathbf{0})\right| \leq Z^{-1} H^{-1}|\tilde{\boldsymbol{f}}(\mathbf{0})| \\
& \leq(A+E)^{-1} H^{-1}|\tilde{\boldsymbol{f}}(\mathbf{0})|=B^{-1} Q H^{-1}|\tilde{\boldsymbol{f}}(\mathbf{0})|
\end{aligned}
$$

where we put

$$
|\tilde{\boldsymbol{f}}(\mathbf{0})|=\left(\begin{array}{c}
\left|f\left(x_{0}, 0,-\frac{\alpha}{\alpha_{1}}\right)-\frac{2}{h_{1}} \frac{\alpha}{\alpha_{1}} p_{0}\right| \\
\left|f\left(x_{1}, 0,0\right)\right| \\
\vdots \\
\left|f\left(x_{n}, 0,0\right)\right| \\
\left|f\left(x_{n+1}, 0, \frac{\beta}{\beta_{1}}\right)-\frac{2}{h_{n+1}} \frac{\beta}{\beta_{1}} p_{n+1}\right|
\end{array}\right)
$$


and used the well known fact that

$$
0<Z^{-1} \leq(A+E)^{-1}=B^{-1} Q
$$

since $Z$ as well as $A+E$ is an irreducibly diagonally dominant $L$-matrix, hence an $M$-matrix whose inverse is a positive matrix (cf. [12]).

It now follows from (4.4) and Lemmas 4.1 and 4.2 that

$$
\begin{aligned}
|\boldsymbol{W}| & \leq e^{7 M \int_{a}^{b} \frac{d t}{p(t)}} A^{-1} Q H^{-1}|\tilde{\boldsymbol{f}}(\mathbf{0})| \\
& \leq e^{7 M \int_{a}^{b} \frac{d t}{p(t)}} A^{-1} \cdot e^{\frac{3 M}{2} \int_{a}^{b} \frac{d t}{p(t)}} H^{-1} c_{\infty}\left(\begin{array}{c}
1 \\
\vdots \\
1
\end{array}\right) \\
& =e^{\frac{17 M}{2} \int_{a}^{b} \frac{d t}{p(t)}} c_{\infty} A^{-1} H^{-1}\left(\begin{array}{c}
1 \\
\vdots \\
1
\end{array}\right)
\end{aligned}
$$

where $c_{\infty}=\|\tilde{\boldsymbol{f}}(\mathbf{0})\|_{\infty}$. Hence

$$
\begin{aligned}
\left|W_{i}\right| & \leq c_{\infty} e^{\frac{17 M}{2} \int_{a}^{b} \frac{d t}{p(t)}}\left[\sum_{j=0}^{n+1} G\left(x_{i}, x_{j}\right) \omega_{j}\right] \\
& \leq c_{\infty} e^{\frac{17 M}{2} \int_{a}^{b} \frac{d t}{p(t)}} G\left(x_{i}, x_{i}\right) \sum_{j=0}^{n+1} \omega_{j} \\
& =c_{\infty} e^{\frac{17 M}{2} \int_{a}^{b} \frac{d t}{p(t)}} G\left(x_{i}, x_{i}\right)(b-a)
\end{aligned}
$$

and

$$
\|\boldsymbol{W}\|_{\infty} \leq c_{\infty} e^{\frac{17 M}{2} \int_{a}^{b} \frac{d t}{p(t)}}\left(\max _{a \leq x \leq b} G(x, x)\right)(b-a) \equiv \delta \quad \text { (say) }
$$

Let

$$
\mathscr{B}=\left\{\boldsymbol{U}=\left(U_{0}, \ldots, U_{n+1}\right)^{t} \in \mathbb{R}^{n+2} \mid\|\boldsymbol{U}\|_{\infty} \leq \delta\right\}
$$

and, define a map $T: \mathscr{B} \rightarrow \mathscr{B}$ by $T \boldsymbol{U}=\boldsymbol{W}$ for $\boldsymbol{U} \in \mathscr{B}$, where $\boldsymbol{W}$ is the unique solution of (4.1). Then $T$ is a continuous map of the compact convex subset $\mathscr{B}$ into itself. Therefore, by the Brouwer theorem, $T$ has a fixed point $\boldsymbol{U}$ in $\mathscr{B}$, which is a solution of (3.4). This proves Theorem 4.1.

Q.E.D.

\section{Error Estimates}

Let $\boldsymbol{U}=\left(U_{0}, \ldots, U_{n+1}\right)^{t}$ be the solution for (3.4) and put $\boldsymbol{u}=\left(u_{0}, \ldots, u_{n+1}\right)^{t}$, where $u_{i}=u\left(x_{i}\right)$ and $u(x)$ is the solution for (1.1)-(1.3). In this section, we shall prove

$$
u_{i}-U_{i}=O\left(h^{2}\right) \quad \forall i
$$


under some assumptions on $p$ and $f$ which are stronger than those of Theorem 2.1. We first state the following lemma.

Lemma 5.1. Let $p \in C^{2,1}[a, b]$ and $f(x, u, v)$ satisfy the assumptions of Theorem 2.1. Furthermore, let $f_{x}$ exist on $\widetilde{\mathscr{R}}=[a, b] \times\left[-\delta_{0}, \delta_{0}\right] \times\left[-\delta_{1}, \delta_{1}\right]$ and $f, f_{x}$, $f_{u}$ and $f_{v}$ satisfy there Lipschitz conditions in $x, u$ and $v$, where $\delta_{0}$ and $\delta_{1}$ are constants defined in (2.7) and (2.8). Then $u \in C^{3,1}[a, b]$.

Proof. Since $p \geq p_{*}=\min _{a \leq x \leq b} p(x)>0, u \in\left[-\delta_{0}, \delta_{0}\right], u^{\prime} \in\left[-\delta_{1}, \delta_{1}\right]$ and

$$
u^{\prime \prime}(x)=\frac{1}{p(x)}\left[f\left(x, u(x), u^{\prime}(x)\right)-p^{\prime}(x) u^{\prime}(x)\right],
$$

we have $u \in C^{2,1}[a, b]$ if $p \in C^{1,1}[a, b]$ and if $f$ satisfies Lipschitz condition on $\widetilde{\mathscr{R}}$. Observe that the right-hand side of (5.1) is differentiable with respect to $x$. Therefore, differentiating both sides of (5.1) with respect to $x$, we obtain

$$
\begin{gathered}
u^{\prime \prime \prime}(x)=\frac{1}{p(x)}\left[f_{x}\left(x, u(x), u^{\prime}(x)\right)+f_{u}\left(x, u(x), u^{\prime}(x)\right) u^{\prime}(x)\right. \\
\left.+f_{u^{\prime}}\left(x, u(x), u^{\prime}(x)\right) u^{\prime \prime}(x)-p^{\prime \prime}(x) u^{\prime}(x)-p^{\prime}(x) u^{\prime \prime}(x)\right] \\
-\frac{p^{\prime}(x)}{p(x)^{2}}\left[f\left(x, u(x), u^{\prime}(x)\right)-p^{\prime}(x) u^{\prime}(x)\right] .
\end{gathered}
$$

Hence, if $p \in C^{2,1}[a, b]$ and $f, f_{x}, f_{u}, f_{u^{\prime}}$ satisfy Lipschitz conditions on $\widetilde{\mathscr{R}}=$ $[a, b] \times\left[-\delta_{0}, \delta_{0}\right] \times\left[-\delta_{1}, \delta_{1}\right]$, then the right-hand side of (5.2) satisfies a Lipschitz condition in $x$ on $[a, b]$ so that we have $u \in C^{3,1}[a, b]$.

Q.E.D.

We denote by $\tau_{i}$ the local truncation error at $x_{i}$ for (3.4) and put $p_{i+\frac{1}{2}}=$ $p\left(x_{i+\frac{1}{2}}\right)=p\left(x_{i}+\frac{1}{2} h_{i+1}\right), i \geq 0$. Then, under the assumption $u \in C^{3,1}[a, b]$, we have

$$
\begin{aligned}
\tau_{0} & =\frac{1}{\omega_{0}}\left[\left(a_{0}+a_{1}\right) u_{0}-a_{1} u_{1}\right]+f\left(x_{0}, u_{0}, \frac{\alpha_{0} u_{0}-\alpha}{\alpha_{1}}\right)-\frac{2}{h_{1}} \frac{\alpha}{\alpha_{1}} p_{0} \\
& =\frac{1}{\omega_{0}}\left[\left(a_{0}+a_{1}\right) u_{0}-a_{1} u_{1}\right]+f\left(x_{0}, u_{0}, u_{0}^{\prime}\right)-\frac{2}{h_{1}} \frac{\alpha}{\alpha_{1}} p_{0} \\
& =\frac{1}{\omega_{0}}\left[\left(a_{0}+a_{1}\right) u_{0}-a_{1} u_{1}\right]+\left[\left(p u^{\prime}\right)^{\prime}\right]_{0}-\frac{2}{h_{1}} \frac{\alpha}{\alpha_{1}} p_{0} \\
& =\frac{2}{h_{1}}\left[\left(\frac{\alpha_{0}}{\alpha_{1}} p_{0}+\frac{1}{\int_{x_{0}}^{x_{1}} \frac{d t}{p(t)}}\right) u_{0}-\frac{1}{\int_{x_{0}}^{x_{1}} \frac{d t}{p(t)}} u_{1}\right]+p_{0} u_{0}^{\prime \prime}+p_{0}^{\prime} u_{0}^{\prime}-\frac{2}{h_{1}} \frac{\alpha}{\alpha_{1}} p_{0} \\
& =\frac{2}{h_{1}}\left[p_{0} u_{0}^{\prime}-\frac{u_{1}-u_{0}}{\int_{x_{0}}^{x_{1}} \frac{d t}{p(t)}}\right]+p_{0} u_{0}^{\prime \prime}+p_{0}^{\prime} u_{0}^{\prime} \\
& =\frac{2}{h_{1}}\left[p_{0} u_{0}^{\prime}-\frac{h_{1} u_{0}^{\prime}+\frac{h_{1}^{2}}{2} u_{0}^{\prime \prime}+O\left(h_{1}^{3}\right)}{\frac{h_{1}}{p_{\frac{1}{2}}}+O\left(h_{1}^{3}\right)}\right]+p_{0} u_{0}^{\prime \prime}+p_{0}^{\prime} u_{0}^{\prime}
\end{aligned}
$$




$$
\begin{aligned}
& =\frac{2}{h_{1}}\left[p_{0} u_{0}^{\prime}-\left(p_{\frac{1}{2}}+O\left(h_{1}^{2}\right)\right)\left(u_{0}^{\prime}+\frac{h_{1}}{2} u_{0}^{\prime \prime}+O\left(h_{1}^{2}\right)\right)\right]+p_{0} u_{0}^{\prime \prime}+p_{0}^{\prime} u_{0}^{\prime} \\
& =\frac{2}{h_{1}}\left[p_{0} u_{0}^{\prime}-\left(p_{0}+\frac{h_{1}}{2} p_{0}^{\prime}+O\left(h_{1}^{2}\right)\right)\left(u_{0}^{\prime}+\frac{h_{1}}{2} u_{0}^{\prime \prime}+O\left(h_{1}^{2}\right)\right)\right]+p_{0} u_{0}^{\prime \prime}+p_{0}^{\prime} u_{0}^{\prime} \\
& =\frac{2}{h_{1}}\left[-\frac{h_{1}}{2} p_{0}^{\prime} u_{0}^{\prime}-\frac{h_{1}}{2} p_{0} u_{0}^{\prime \prime}+O\left(h_{1}^{2}\right)\right]+p_{0} u_{0}^{\prime \prime}+p_{0}^{\prime} u_{0}^{\prime} \\
& =O\left(h_{1}\right) .
\end{aligned}
$$

Similarly, we obtain

$$
\begin{aligned}
\tau_{n+1}= & \frac{1}{\omega_{n+1}}\left[-a_{n+1} u_{n}+\left(a_{n+1}+a_{n+2}\right) u_{n+1}\right] \\
& +f\left(x_{n+1}, u_{n+1}, \frac{\beta-\beta_{0} u_{n+1}}{\beta_{1}}\right)-\frac{2}{h_{n+1}} \frac{\beta}{\beta_{1}} p_{n+1} \\
= & O\left(h_{n+1}\right) .
\end{aligned}
$$

In order to estimate $\tau_{i}, 1 \leq i \leq n$, we prove the following three lemmas.

LEMMA 5.2. Let

$$
\gamma_{i}=p_{i+\frac{1}{2}} \frac{u_{i+1}-u_{i}}{h_{i+1}}+p_{i-\frac{1}{2}} \frac{u_{i-1}-u_{i}}{h_{i}} .
$$

If $p \in C^{2,1}[a, b]$ and $u \in C^{3,1}[a, b]$, then

$$
\frac{2}{h_{i+1}+h_{i}} \gamma_{i}=p_{i} u_{i}^{\prime \prime}+p_{i}^{\prime} u_{i}^{\prime}+\left(h_{i+1}-h_{i}\right) \kappa_{i}+O\left(h^{2}\right),
$$

where

$$
\kappa(x)=\frac{1}{12}\left\{3 p^{\prime \prime}(x) u^{\prime}(x)+6 p^{\prime}(x) u^{\prime \prime}(x)+4 p(x) u^{\prime \prime \prime}(x)\right\}
$$

and

$$
\kappa_{i}=\kappa\left(x_{i}\right)
$$

Proof. We have

$$
\begin{aligned}
\gamma_{i}=\left\{p_{i}+\right. & \left.\frac{h_{i+1}}{2} p_{i}^{\prime}+\frac{1}{2}\left(\frac{h_{i+1}}{2}\right)^{2} p_{i}^{\prime \prime}+O\left(h_{i+1}^{3}\right)\right\} \\
& \times\left\{u_{i}^{\prime}+\frac{1}{2} h_{i+1} u_{i}^{\prime \prime}+\frac{1}{6} h_{i+1}^{2} u_{i}^{\prime \prime \prime}+O\left(h_{i+1}^{3}\right)\right\} \\
+ & \left\{p_{i}-\frac{h_{i}}{2} p_{i}^{\prime}+\frac{1}{2}\left(\frac{h_{i}}{2}\right)^{2} p_{i}^{\prime \prime}+O\left(h_{i}^{3}\right)\right\} \\
& \times\left\{-u_{i}^{\prime}+\frac{1}{2} h_{i} u_{i}^{\prime \prime}-\frac{1}{6} h_{i}^{2} u_{i}^{\prime \prime \prime}+O\left(h_{i}^{3}\right)\right\}
\end{aligned}
$$




$$
\begin{gathered}
=\frac{h_{i+1}+h_{i}}{2}\left[\left\{p_{i}^{\prime}+\frac{1}{4}\left(h_{i+1}-h_{i}\right) p_{i}^{\prime \prime}\right\} u_{i}^{\prime}+\left\{p_{i}+\frac{1}{2}\left(h_{i+1}-h_{i}\right) p_{i}^{\prime}\right\} u_{i}^{\prime \prime}\right. \\
\left.+\frac{1}{3}\left(h_{i+1}-h_{i}\right) p_{i} u_{i}^{\prime \prime \prime}\right]+O\left(h_{i+1}^{3}\right)+O\left(h_{i}^{3}\right) .
\end{gathered}
$$

Hence

$$
\begin{aligned}
\frac{2}{h_{i+1}+h_{i}} \gamma_{i}= & p_{i}^{\prime} u_{i}^{\prime}+p_{i} u_{i}^{\prime \prime} \\
& +\frac{h_{i+1}-h_{i}}{12}\left(3 p_{i}^{\prime \prime} u_{i}^{\prime}+6 p_{i}^{\prime} u_{i}^{\prime \prime}+4 p_{i} u_{i}^{\prime \prime \prime}\right)+O\left(h^{2}\right) .
\end{aligned}
$$

This proves Lemma 5.2.

Q.E.D.

Lemma 5.3. Let $y \in C^{2,1}[-h, h]$. Then

$$
\int_{-h}^{h} y(t) d t=2 h y(0)+\frac{1}{3} y^{\prime \prime}(0) h^{3}+O\left(h^{4}\right) .
$$

Proof. Let

$$
Y(h)=\int_{-h}^{h} y(t) d t-2 h y(0) .
$$

Then

$$
\begin{aligned}
& Y^{\prime}(h)=y(h)+y(-h)-2 y(0), \\
& Y^{\prime \prime}(h)=y^{\prime}(h)-y^{\prime}(-h), \\
& Y^{\prime \prime \prime}(h)=y^{\prime \prime}(h)+y^{\prime \prime}(-h)
\end{aligned}
$$

so that $Y(0)=Y^{\prime}(0)=Y^{\prime \prime}(0)=0, Y^{\prime \prime \prime}(0)=2 y^{\prime \prime}(0)$ and

$$
\begin{aligned}
Y(h) & =\frac{1}{3 !} Y^{\prime \prime \prime}(0) h^{3}+\frac{1}{3 !}\left(Y^{\prime \prime \prime}(\theta h)-Y^{\prime \prime \prime}(0)\right) h^{3} \\
& =\frac{1}{3} y^{\prime \prime}(0) h^{3}+O\left(h^{4}\right) .
\end{aligned}
$$

Q.E.D.

Lemma 5.4. Let $a_{i}$ be defined as in (3.1) and set

$$
\varepsilon_{i}=\left(\frac{p_{i+\frac{1}{2}}}{h_{i+1}}-a_{i+1}\right)\left(u_{i+1}-u_{i}\right)+\left(\frac{p_{i-\frac{1}{2}}}{h_{i}}-a_{i}\right)\left(u_{i-1}-u_{i}\right) .
$$

Then we have

$$
\varepsilon_{i}=\left(s_{i+\frac{1}{2}} h_{i+1}^{2}-s_{i-\frac{1}{2}} h_{i}^{2}\right) u_{i}^{\prime}+O\left(h_{i+1}^{3}\right)+O\left(h_{i}^{3}\right),
$$

provided that $u \in C^{3,1}[a, b]$, where

$$
s(x)=\frac{1}{24}\left(\frac{1}{p(x)}\right)^{\prime \prime} p(x)^{2} .
$$


Proof. By Lemma 5.3, we have

$$
\begin{aligned}
& \left(\frac{p_{i+\frac{1}{2}}}{h_{i+1}}-a_{i+1}\right)\left(u_{i+1}-u_{i}\right) \\
& =\left\{\frac{p_{i+\frac{1}{2}}}{h_{i+1}}-\frac{1}{\frac{h_{i+1}}{p_{i+\frac{1}{2}}}+\frac{1}{24}\left(\frac{1}{p}\right)_{i+\frac{1}{2}}^{\prime \prime} h_{i+1}^{3}+O\left(h_{i+1}^{4}\right)}\right\} \\
& \times\left(u_{i}^{\prime} h_{i+1}+\frac{1}{2} u_{i}^{\prime \prime} h_{i+1}^{2}+O\left(h_{i+1}^{3}\right)\right) \\
& =\left\{\frac{p_{i+\frac{1}{2}}}{h_{i+1}}-\frac{1}{\frac{h_{i+1}}{p_{i+\frac{1}{2}}}\left(1+\frac{1}{24}\left(\frac{1}{p}\right)_{i+\frac{1}{2}}^{\prime \prime} h_{i+1}^{2} p_{i+\frac{1}{2}}+O\left(h_{i+1}^{3}\right)\right)}\right\} \\
& \times\left(u_{i}^{\prime} h_{i+1}+\frac{1}{2} u_{i}^{\prime \prime} h_{i+1}^{2}+O\left(h_{i+1}^{3}\right)\right) \\
& =\left\{\frac{p_{i+\frac{1}{2}}}{h_{i+1}}-\frac{p_{i+\frac{1}{2}}}{h_{i+1}}\left(1-\frac{1}{24}\left(\frac{1}{p}\right)_{i+\frac{1}{2}}^{\prime \prime} h_{i+1}^{2} p_{i+\frac{1}{2}}+O\left(h_{i+1}^{3}\right)\right)\right\} \\
& \times\left(u_{i}^{\prime} h_{i+1}+\frac{1}{2} u_{i}^{\prime \prime} h_{i+1}^{2}+O\left(h_{i+1}^{3}\right)\right) \\
& =\left\{\frac{1}{24}\left(\frac{1}{p}\right)_{i+\frac{1}{2}}^{\prime \prime} p_{i+\frac{1}{2}}^{2} h_{i+1} u_{i}^{\prime}+O\left(h_{i+1}^{2}\right)\right\}\left(u_{i}^{\prime} h_{i+1}+\frac{1}{2} u_{i}^{\prime \prime} h_{i+1}^{2}+O\left(h_{i+1}^{3}\right)\right) \\
& =\frac{1}{24}\left(\frac{1}{p}\right)_{i+\frac{1}{2}}^{\prime \prime} p_{i+\frac{1}{2}}^{2} h_{i+1}^{2} u_{i}^{\prime}+O\left(h_{i+1}^{3}\right)
\end{aligned}
$$

and, similarly,

$$
\left(\frac{p_{i-\frac{1}{2}}}{h_{i}}-a_{i}\right)\left(u_{i-1}-u_{i}\right)=-\frac{1}{24}\left(\frac{1}{p}\right)_{i-\frac{1}{2}}^{\prime \prime} p_{i-\frac{1}{2}}^{2} h_{i}^{2} u_{i}^{\prime}+O\left(h_{i}^{3}\right) .
$$

Hence we obtain

$$
\varepsilon_{i}=\left(s_{i+\frac{1}{2}} h_{i+1}^{2}-s_{i-\frac{1}{2}} h_{i}^{2}\right) u_{i}^{\prime}+O\left(h_{i+1}^{3}\right)+O\left(h_{i}^{3}\right), 1 \leq i \leq n
$$

with $s(x)=\frac{1}{24}\left(\frac{1}{p}\right)^{\prime \prime} p^{2}$.

By Lemmas 5.2-5.4, we have for $1 \leq i \leq n$

$$
\begin{aligned}
\tau_{i} \equiv & \frac{2}{h_{i+1}+h_{i}}\left[-a_{i+1}\left(u_{i+1}-u_{i}\right)-a_{i}\left(u_{i-1}-u_{i}\right)\right] \\
& +f\left(x_{i}, u_{i}, \frac{u_{i+1}-u_{i-1}}{h_{i+1}+h_{i}}\right)
\end{aligned}
$$




$$
\begin{aligned}
= & \frac{2}{h_{i+1}+h_{i}}\left[\varepsilon_{i}-\gamma_{i}\right]+f\left(x_{i}, u_{i}, \frac{u_{i+1}-u_{i-1}}{h_{i+1}+h_{i}}\right) \\
= & \frac{2}{h_{i+1}+h_{i}} \varepsilon_{i}-\left\{p_{i} u_{i}^{\prime \prime}+p_{i}^{\prime} u_{i}^{\prime}+\left(h_{i+1}-h_{i}\right) \kappa_{i}+O\left(h^{2}\right)\right\} \\
& \quad+f\left(x_{i}, u_{i}, \frac{u_{i+1}-u_{i-1}}{h_{i+1}+h_{i}}\right) \\
= & \frac{2}{h_{i+1}+h_{i}}\left(s_{i+\frac{1}{2}} h_{i+1}^{2}-s_{i-\frac{1}{2}} h_{i}^{2}\right) u_{i}^{\prime}+O\left(h^{2}\right)-\left(h_{i+1}-h_{i}\right) \kappa_{i}+O\left(h^{2}\right) \\
& +f\left(x_{i}, u_{i}, \frac{u_{i+1}-u_{i-1}}{h_{i+1}+h_{i}}\right)-f\left(x_{i}, u_{i}, u_{i}^{\prime}\right) \\
= & \frac{2}{h_{i+1}+h_{i}}\left(s_{i+\frac{1}{2}} h_{i+1}^{2}-s_{i-\frac{1}{2}} h_{i}^{2}\right) u_{i}^{\prime}-\left(h_{i+1}-h_{i}\right) \kappa_{i}+O\left(h^{2}\right) \\
& +\left\{\frac{1}{2}\left(h_{i+1}-h_{i}\right) u_{i}^{\prime \prime}+O\left(h^{2}\right)\right\} q_{i},
\end{aligned}
$$

where

$$
q_{i}=\int_{0}^{1} \frac{\partial f}{\partial u^{\prime}}\left(x_{i}, u_{i}, u_{i}^{\prime}+\theta\left(\frac{u_{i+1}-u_{i-1}}{h_{i+1}+h_{i}}-u_{i}^{\prime}\right)\right) d \theta .
$$

Let $\boldsymbol{\tau}=\left(\tau_{0}, \ldots, \tau_{n+1}\right)^{t}$ and $\boldsymbol{W}=\boldsymbol{u}-\boldsymbol{U}=\left(W_{0}, \ldots, W_{n+1}\right)^{t}$. Then

$$
H A \boldsymbol{u}+\widetilde{\boldsymbol{f}}(\boldsymbol{u})=\boldsymbol{\tau}
$$

and

$$
H A \boldsymbol{W}+\tilde{\boldsymbol{f}}(\boldsymbol{u})-\tilde{\boldsymbol{f}}(\boldsymbol{U})=\boldsymbol{\tau} .
$$

Furthermore, we write

$$
\widetilde{\boldsymbol{f}}(\boldsymbol{u})-\widetilde{\boldsymbol{f}}(\boldsymbol{U})=(\widetilde{D}+H \widetilde{E}) \boldsymbol{W}
$$

with a diagonal matrix $\widetilde{D}=\left(\widetilde{d}_{0}, \widetilde{d}_{1}, \ldots, \widetilde{d}_{n+1}\right)$ and a tridiagonal matrix

$$
\widetilde{E}=\left(\begin{array}{ccccc}
\widetilde{\sigma}_{0} & 0 & & & \\
-\widetilde{\sigma}_{1} & 0 & \widetilde{\sigma}_{1} & & \\
\ddots & \ddots & \ddots & & \\
& & -\widetilde{\sigma}_{n} & 0 & \widetilde{\sigma}_{n} \\
& & & 0 & \widetilde{\sigma}_{n+1}
\end{array}\right)
$$

whose elements $\widetilde{d}_{i}$ and $\widetilde{\sigma}_{i}$ are defined as follows:

$$
\begin{aligned}
\widetilde{d}_{0} & =\int_{0}^{1} \frac{\partial f}{\partial u}\left(x_{0}, U_{0}+\theta W_{1}, \frac{\alpha_{0}\left(U_{0}+\theta W_{0}\right)-\alpha}{\alpha_{1}}\right) d \theta, \\
\widetilde{d}_{i}=\int_{0}^{1} \frac{\partial f}{\partial u}\left(x_{i}, U_{i}+\theta W_{i}, \frac{1}{h_{i+1}+h_{i}}\left(U_{i+1}-U_{i-1}+\theta\left(W_{i+1}-W_{i-1}\right)\right)\right) d \theta & 1 \leq i \leq n,
\end{aligned}
$$




$$
\begin{aligned}
& \widetilde{d}_{n+1}=\int_{0}^{1} \frac{\partial f}{\partial u}\left(x_{n+1}, U_{n+1}+\theta W_{n+1}, \frac{\beta-\beta_{0}\left(U_{n+1}+\theta W_{n+1}\right)}{\beta_{1}}\right) d \theta \\
& \widetilde{\sigma_{0}}=\omega_{0} \frac{\alpha_{0}}{\alpha_{1}} \int_{0}^{1} \frac{\partial f}{\partial u^{\prime}}\left(x_{0}, U_{0}+\theta W_{0}, \frac{\alpha_{0}\left(U_{0}+\theta W_{0}\right)-\alpha}{\alpha_{1}}\right) d \theta \\
& \widetilde{\sigma}_{i}=\frac{1}{2} \int_{0}^{1} \frac{\partial f}{\partial u^{\prime}}\left(x_{i}, U_{i}+\theta W_{i}, \frac{1}{h_{i+1}+h_{i}}\left(U_{i+1}-U_{i-1}+\theta\left(W_{i+1}-W_{i-1}\right)\right)\right) d \theta \\
& \widetilde{\sigma}_{n+1}=-\omega_{n+1} \frac{\beta_{0}}{\beta_{1}} \int_{0}^{1} \frac{\partial f}{\partial u^{\prime}}\left(x_{n+1}, U_{n+1}+\theta W_{n+1}, \frac{\beta-\beta_{0}\left(U_{n+1}+\theta W_{n+1}\right)}{\beta_{1}}\right) d \theta .
\end{aligned}
$$

Hence we have from (5.7)

$$
H\left(A+\widetilde{E}+H^{-1} \widetilde{D}\right) \boldsymbol{W}=\boldsymbol{\tau}
$$

and

$$
\begin{aligned}
\boldsymbol{W} & =\left(A+\widetilde{E}+H^{-1} \widetilde{D}\right)^{-1} H^{-1} \boldsymbol{\tau} \\
& =\left[(A+\widetilde{E})^{-1}-\left(A+\widetilde{E}+H^{-1} \widetilde{D}\right)^{-1} H^{-1} \widetilde{D}(A+\widetilde{E})^{-1}\right] H^{-1} \boldsymbol{\tau} \\
& =\widetilde{A}^{-1} H^{-1} \boldsymbol{\tau}-\left(\widetilde{A}+H^{-1} \widetilde{D}\right)^{-1} H^{-1} \widetilde{D} \widetilde{A}^{-1} H^{-1} \boldsymbol{\tau}
\end{aligned}
$$

where we put $\widetilde{A}=A+\widetilde{E}$.

Next, we shall estimate $\widetilde{A}^{-1}=\left(\widetilde{\alpha}_{i j}\right)$. As in $\widetilde{\beta}_{4}$, we choose a diagonal matrix $\widetilde{Q}=\operatorname{diag}\left(\widetilde{\rho}_{0}, \widetilde{\rho}_{1}, \ldots, \widetilde{\rho}_{n+1}\right)$ with $\widetilde{\rho}_{0}=1$ so that $\widetilde{B}=\widetilde{Q} \widetilde{A}$ is symmetric. We then have

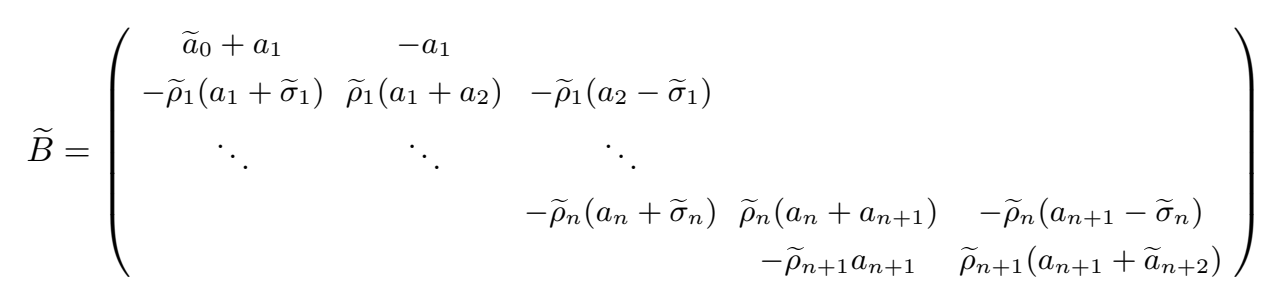

where

$$
\widetilde{a}_{0}=a_{0}+\widetilde{\sigma}_{0}, \quad \widetilde{a}_{n+2}=a_{n+2}+\widetilde{\sigma}_{n+1} .
$$

Hence, an application of the inversion formula [14] to $\widetilde{B}$ yields that $\widetilde{B}^{-1}=\left(\widetilde{\beta}_{i j}\right)$ is given by

$$
\widetilde{\beta}_{i j}= \begin{cases}\frac{1}{\widetilde{z}_{n+2}} \widetilde{z}_{i}\left(\widetilde{z}_{n+2}-\widetilde{z}_{j}\right) & (i \leq j) \\ \frac{1}{\widetilde{z}_{n+2}}\left(\widetilde{z}_{n+2}-\widetilde{z}_{i}\right) \widetilde{z}_{j} & (i \geq j)\end{cases}
$$


where

$$
\widetilde{z}_{i}= \begin{cases}\frac{1}{\widetilde{a}_{0}} & (i=0) \\ \widetilde{z}_{i-1}+\frac{1}{\widetilde{\rho}_{i}\left(a_{i}+\widetilde{\sigma}_{i}\right)} & (1 \leq i \leq n) \\ \widetilde{z}_{n}+\frac{1}{\widetilde{\rho}_{n+1} a_{n+1}} & (i=n+1) \\ \widetilde{z}_{n+1}+\frac{1}{\widetilde{\rho}_{n+1} \widetilde{a}_{n+2}} & (i=n+2) .\end{cases}
$$

LEMMA 5.5. If $h$ is sufficiently small, then

$$
e^{-\frac{3 M}{2} \int_{a}^{b} \frac{d t}{p(t)}}<\widetilde{\rho}_{i}<e^{\frac{3 M}{2} \int_{a}^{b} \frac{d t}{p(t)}}, \quad 0 \leq i \leq n+1
$$

and

$$
\widetilde{B}^{-1}<e^{7 M \int_{a}^{b} \frac{d t}{p(t)}} \cdot A^{-1}
$$

Proof. The same proof as in Lemma 4.1 works.

Q.E.D.

We are now in a position to prove the following result.

THEOREM 5.1. In addition to the assumptions of Lemma 5.1, assume that $f_{v}(x, u, v)$ satisfies a Lipschitz condition in $x, u, v$ on $\widehat{\mathscr{R}}=[a, b] \times\left[-\delta_{0}, \delta_{0}\right] \times[-(1+$ $\left.\varepsilon) \delta_{1},(1+\varepsilon) \delta_{1}\right]$, where $\varepsilon$ is a positive number which may be chosen arbitrarily small. Then

$$
u_{i}-U_{i}=O\left(h^{2}\right), \quad 0 \leq i \leq n+1 .
$$

Proof. We first remark that $u \in C^{3,1}[a, b]$ by Lemma 5.1. We have from $(5.3)-(5.5)$

$$
\begin{aligned}
\left(\widetilde{A}^{-1} H^{-1} \boldsymbol{\tau}\right)_{i}= & \left(\widetilde{B}^{-1} \widetilde{Q} H^{-1} \boldsymbol{\tau}\right)_{i} \\
= & \sum_{j=0}^{n+1} \widetilde{\beta}_{i j} \widetilde{\rho}_{j} \omega_{j} \tau_{j} \\
= & \widetilde{\beta}_{i 0} \frac{h_{1}}{2} O\left(h_{1}\right)+\widetilde{\beta}_{i n+1} \widetilde{\rho}_{n+1} \frac{h_{n+1}}{2} O\left(h_{n+1}\right) \\
& +\sum_{j=1}^{n} \widetilde{\beta}_{i j} \widetilde{\rho}_{j}\left[\left(s_{j+\frac{1}{2}} h_{j+1}^{2}-s_{j-\frac{1}{2}} h_{j}^{2}\right) u_{j}^{\prime}\right. \\
& \left.\quad-\frac{1}{2}\left(h_{j+1}^{2}-h_{j}^{2}\right)\left(\kappa_{j}-\frac{1}{2} u_{j}^{\prime \prime} q_{j}\right)+O\left(h^{3}\right)\right] \\
= & \sum_{j=1}^{n}\left(\varphi_{i}{ }_{j+1} h_{j+1}^{2}-\psi_{i j} h_{j}^{2}\right)+O\left(h^{2}\right) \\
= & \sum_{j=2}^{n}\left(\varphi_{i j}-\psi_{i j}\right) h_{j}^{2}+\varphi_{i n+1} h_{n+1}^{2}-\psi_{i 1} h_{1}^{2}+O\left(h^{2}\right),
\end{aligned}
$$




$$
=\sum_{j=2}^{n}\left(\varphi_{i j}-\psi_{i j}\right) h_{j}^{2}+O\left(h^{2}\right)
$$

where

$$
\begin{aligned}
\varphi_{i j} & =\widetilde{\beta}_{i j-1} \widetilde{\rho}_{j-1}\left[s_{j-\frac{1}{2}} u_{j-1}^{\prime}-\frac{1}{2}\left(\kappa_{j-1}-\frac{1}{2} u_{j-1}^{\prime \prime} q_{j-1}\right)\right] \\
\psi_{i j} & =\widetilde{\beta}_{i j} \widetilde{\rho}_{j}\left[s_{j-\frac{1}{2}} u_{j}^{\prime}-\frac{1}{2}\left(\kappa_{j}-\frac{1}{2} u_{j}^{\prime \prime} q_{j}\right)\right] .
\end{aligned}
$$

We then have from (5.10)

$$
\widetilde{\beta}_{i j}-\widetilde{\beta}_{i j-1}=O(h) \quad \forall i, j .
$$

In fact, if $i \leq j-1$, for example, then

$$
z_{j}-z_{j-1}=\frac{1}{\widetilde{\rho}_{j}\left(a_{j}+\widetilde{\sigma}_{j}\right)}<\frac{1}{a_{j}} \cdot \frac{1}{\widetilde{\rho}_{j}} \cdot \frac{1}{1-\frac{M}{2 a_{j}}}=O\left(\frac{1}{a_{j}}\right)=O\left(h_{j}\right)
$$

since $\frac{1}{\widetilde{\rho}_{j}}=O(1)$ by Lemma 5.5, and

$$
\begin{aligned}
\left|\widetilde{\beta}_{i j}-\widetilde{\beta}_{i j-1}\right| & =\left|\frac{1}{\widetilde{z}_{n+2}} \widetilde{z}_{i}\left(\widetilde{z}_{j-1}-\widetilde{z}_{j}\right)\right| \\
& <\left|\widetilde{z}_{j-1}-\widetilde{z}_{j}\right|=O\left(h_{j}\right) \text { for } j \geq 1
\end{aligned}
$$

We also have $\widetilde{\rho}_{j}-\widetilde{\rho}_{j-1}=O(h)$ for $2 \leq j \leq n$. In fact, we have

$$
\begin{aligned}
& \widetilde{\rho}_{1}=\frac{a_{1}}{a_{1}+\widetilde{\sigma}_{1}}=O(1) \\
& \widetilde{\rho}_{2}-\widetilde{\rho}_{1}=\frac{a_{2}-\widetilde{\sigma}_{1}}{a_{2}+\widetilde{\sigma}_{2}} \widetilde{\rho}_{1}-\widetilde{\rho}_{1}=-\frac{\widetilde{\sigma}_{1}+\widetilde{\sigma}_{2}}{a_{2}+\widetilde{\sigma}_{2}} \widetilde{\rho}_{1}=O\left(\frac{1}{a_{2}}\right)=O\left(h_{2}\right), \text { etc. }
\end{aligned}
$$

Since $p \in C^{2,1}[a, b]$ and $u \in C^{3,1}[a, b]$, we have

$$
\begin{gathered}
p_{j}^{(k-1)}-p_{j-1}^{(k-1)}=O(h), u_{j}^{(k)}-u_{j-1}^{(k)}=O(h), k=1,2,3, \\
\text { and } \kappa_{j}-\kappa_{j-1}=O(h) .
\end{gathered}
$$

Furthermore,

$$
q_{j}-q_{j-1}=O(h)
$$

since, in (5.6),

$$
u_{i}^{\prime} \in\left[-\delta_{1}, \delta_{1}\right] \text { and } \frac{u_{i+1}-u_{i-1}}{h_{i+1}+h_{i}}-u_{i}^{\prime}=O(h) \quad \forall i
$$


so that, given $\varepsilon>0$,

$$
u_{i}^{\prime}+\theta\left(\frac{u_{i+1}-u_{i-1}}{h_{i+1}+h_{i}}-u_{i}^{\prime}\right) \in\left[-(1+\varepsilon) \delta_{1},(1+\varepsilon) \delta_{1}\right] \quad \forall i
$$

for sufficiently small $h$. Therefore, we have

$$
\varphi_{i j}-\psi_{i j}=O(h)
$$

Consequently, we obtain

$$
\sum_{j=2}^{n}\left(\varphi_{i j}-\psi_{i j}\right) h_{j}^{2}=O\left(h^{2}\right)
$$

and, from (5.12),

$$
\left(\widetilde{A}^{-1} H^{-1} \boldsymbol{\tau}\right)_{i}=O\left(h^{2}\right) .
$$

On the other hand, it follows from (5.9) that

$$
\begin{aligned}
|\boldsymbol{W}| & \leq\left|\widetilde{A}^{-1} H^{-1} \boldsymbol{\tau}\right|+\left(\widetilde{A}+H^{-1} \widetilde{D}\right)^{-1} H^{-1} \widetilde{D}\left|\widetilde{A}^{-1} H^{-1} \boldsymbol{\tau}\right| \\
& \leq\left|\widetilde{A}^{-1} H^{-1} \boldsymbol{\tau}\right|+\widetilde{A}^{-1} H^{-1} \widetilde{D}\left|\widetilde{A}^{-1} H^{-1} \boldsymbol{\tau}\right|
\end{aligned}
$$

Furthermore, by Lemma 5.5

$$
\widetilde{A}^{-1}=\widetilde{B}^{-1} \widetilde{Q}<e^{\frac{17 M}{2} \int_{a}^{b} \frac{d t}{p(t)}} A^{-1}=e^{\frac{17 M}{2} \int_{a}^{b} \frac{d t}{p(t)}}\left(G\left(x_{i}, x_{j}\right)\right)
$$

and

$$
\widetilde{D} \leq K I
$$

where $K$ is the constant defined in (2.4). We thus conclude

$$
W_{i}=u_{i}-U_{i}=O\left(h^{2}\right) \quad \forall i
$$

from (5.13)-(5.16).

Q.E.D.

REMARK 5.1. If $f=f(x, u)$, then Remark 2.1 applies to Theorem 5.1, too.

\section{Observations}

In (3.4), if we approximate $\int_{x_{i-1}}^{x_{i}} \frac{d t}{p(t)}$ by the midpoint rule, then the $i$ th equation reduces to the well known formula

$$
\begin{array}{r}
-\left[\frac{p_{i+\frac{1}{2}} \frac{U_{i+1}-U_{i}}{h_{i+1}}-p_{i-\frac{1}{2}} \frac{U_{i}-U_{i-1}}{h_{i}}}{\frac{h_{i+1}+h_{i}}{2}}\right]+f\left(x_{i}, U_{i}, \frac{U_{i+1}-U_{i-1}}{h_{i+1}+h_{i}}\right)=0 \\
1 \leq i \leq n .
\end{array}
$$


Furthermore, if the boundary conditions (1.2) and (1.3) are approximated by the fictitious node method ( $c f$. Allen-Isaacson [2], pp. 407-408), then

$$
\begin{aligned}
& \frac{2}{h_{1}}\left\{\left(\frac{\alpha_{0}}{\alpha_{1}} p_{0}-\frac{\alpha_{0}}{2 \alpha_{1}} p_{0}^{\prime} h_{1}+\frac{p_{0}}{h_{1}}\right) U_{0}-\frac{p_{0}}{h_{1}} U_{1}\right\} \\
& \quad+f\left(x_{0}, U_{0}, \frac{\alpha_{0} U_{0}-\alpha}{\alpha_{1}}\right)+\frac{\alpha}{\alpha_{1}}\left(p_{0}^{\prime}-\frac{2}{h_{1}} p_{0}\right)=0
\end{aligned}
$$

and

$$
\begin{aligned}
& \frac{2}{h_{n+1}}\left\{-\frac{p_{n+1}}{h_{n+1}} U_{n}+\left(\frac{p_{n+1}}{h_{n+1}}+\frac{\beta_{0}}{\beta_{1}} p_{n+1}+\frac{\beta_{0}}{2 \beta_{1}} p_{n+1}^{\prime} h_{n+1}\right) U_{n+1}\right\} \\
& \quad+f\left(x_{n+1}, U_{n+1}, \frac{\beta-\beta_{0} U_{n+1}}{\beta_{1}}\right)-\frac{\beta}{\beta_{1}}\left(p_{n+1}^{\prime}+\frac{2}{h_{n+1}} p_{n+1}\right)=0 .
\end{aligned}
$$

Hence, we can write (6.1)-(6.3) in the form

$$
H \widehat{A} \boldsymbol{U}+\widehat{\boldsymbol{f}}(\boldsymbol{U})=\mathbf{0}
$$

where

$$
\widehat{A}=\left(\begin{array}{cccc}
\widehat{a}_{0}+\widehat{a}_{1} & -\widehat{a}_{1} & & \\
-\bar{a}_{1} & \bar{a}_{1}+\bar{a}_{2} & -\bar{a}_{2} & \\
\ddots & \ddots & \ddots & \\
& & -\widehat{a}_{n+1} & \widehat{a}_{n+1}+\widehat{a}_{n+2}
\end{array}\right)
$$

with

$$
\begin{aligned}
& \widehat{a}_{0}=a_{0}+O\left(h_{1}\right), \quad \widehat{a}_{1}=a_{1}+O(1), \\
& \bar{a}_{i}=a_{i}+O\left(h_{i}^{2}\right), \quad 1 \leq i \leq n+1, \\
& \widehat{a}_{n+1}=a_{n+1}+O(1), \quad \widehat{a}_{n+2}=a_{n+2}+O\left(h_{n+1}\right)
\end{aligned}
$$

and

$$
\widehat{\boldsymbol{f}}(\boldsymbol{U})=\widetilde{\boldsymbol{f}}(\boldsymbol{U})+\left(\frac{\alpha}{\alpha_{1}} p_{0}^{\prime}, 0, \ldots, 0,-\frac{\beta}{\beta_{1}} p_{n+1}^{\prime}\right)^{t} .
$$

It is interesting to compare (3.4) with (6.4) and observe that, in spite of this difference between $A$ and $\widehat{A}$, the accuracy of the solution of (6.4) is of the second order, too. The proof will be done along the same line as in the proof of Theorem 5.1.

Finally we note that on the basis of an approach developed in [16], numerical methods with fourth order accuracy for solving (1.1)-(1.3) in the case $f=f(x, u)$ are given in Aguchi-Yamamoto [1].

Acknowledgements. The authors are grateful to the referees for helpful comments, especially for pointing out the defect of the original proof of Theorem 2.1. Theorem 5.1 has also been improved. 


\section{References}

[1] S. Aguchi and T. Yamamoto, Numerical methods with fourth order accuracy for two-point boundary value problems. RIMS Kokyuroku, Kyoto Univ., No.1381, 2004, 11-20.

[ 2 ] M.B. Allen and E.L. Isaacson, Numerical Analysis for Applied Science. John Wiley \& Sons, 1998.

[ 3 ] U.M. Ascher, R.M.M. Mattheij and R.D. Russel, Numerical Solution of Boundary Value Problems for Ordinary Differential Equations. Prentice Hall, 1988.

[ 4 ] F.de Hoog and D. Jackett, On the rate of convergence of finite difference schemes on nonuniform grids. J. Austral. Math. Soc. Ser. B, 26 (1985), 247-256.

[ 5 ] P. Henrici, Discrete Variable Methods in Ordinary Differential Equations. Wiley, 1962.

[ 6 ] H.B. Keller, Numerical Methods for Two-Point Boundary Value Problems. Blaisdell, 1968.

[ 7 ] H.O. Kreiss, T.A. Manteuffel, B. Swartz, B. Wendroff and A.B. White, Jr., Supraconvergence schemes on irregular grids. Math. of Comp., 47 (1986), 537-554.

[ 8 ] M. Lees, Discrete methods for nonlinear two-point boundary value problems. Numerical Solution of Partial Differential Equations (ed. J.H. Bramble), Academic Press, 1966, 59-72.

[ 9 ] T.A. Manteuffel and A.B. White, Jr., The numerical solution of a second-order boundary value problems on nonuniform meshes. Math. of Comp., 47 (1986), 511-535.

[10] J. Ortega and W.C. Rheinboldt, Iterative Solution of Nonlinear Equations in Several Variables. Academic Press, 1970 (Reprinted by SIAM, 2000).

[11] A.N. Tikhonov and A.A. Samarskii, Homogeneous difference schemes of a high degree of accuracy of non-uniform nets. U.S.S.R. Comp.Math. \& Math. Phys., 1 (1961), 465-486.

[12] R.S. Varga, Matrix Iterative Analysis. Springer, 2000.

[13] T. Yamamoto, Harmonic relations between Green's functions and Green's matrices for boundary value problems. RIMS Kokyuroku, Kyoto Univ., No.1169, 2000, 15-26.

[14] T. Yamamoto, Inversion formulas for tridiagonal matrices with applications to boundary value problems. Numer. Funct. Anal. \& Optimiz., 22 (2001), 357-385.

[15] T.Yamamoto, Harmonic relations between Green's functions and Green's matrices for boundary value problems II. RIMS Kokyuroku, Kyoto Univ. No.1286, 2002, 27-33.

[16] T.Yamamoto, Harmonic relations between Green's functions and Green's matrices for boundary value problems III. RIMS Kokyuroku, Kyoto Univ. No.1381, 2004, 1-10. 